Diego Paolo Ferruzzo Correa

SYMMETRIC BIFURCATION ANALYSIS OF SYNCHRONOUS STATES OF TIME-DELAY OSCILLATORS NETWORKS

São Paulo

2014 

Diego Paolo Ferruzzo Correa

\section{SYMMETRIC BIFURCATION ANALYSIS OF SYNCHRONOUS STATES OF TIME-DELAY OSCILLATORS NETWORKS}

Text presented to Escola Politécnica da Universidade de São Paulo as a thesis to obtain a $\mathrm{PhD}$ degree in Electrical Engineering.

Specific area: Systems Engineering

Supervisor:

Prof. PhD. José Roberto Castilho Piqueira

São Paulo

2014 


\section{Cataloging-in-Publication Data}

\section{Ferruzzo Correa, Diego Paolo}

Symmetric bifurcation analysis of synchronous states of time-delay oscillators networks / D.P. Ferruzzo Correa. - São Paulo, 2014.

88 p.

Tese (Doutorado) - Escola Politécnica da Universidade de São Paulo. Departamento de Engenharia de Telecomunicações e Controle.

1.Osciladores (Frequência) 2.Sicronização I.Universidade de São Paulo. Escola Politécnica. Departamento de Engenharia de Telecomunicações e Controle IL.t. 



\section{Acknowledgments}

I would like to express my special appreciation and thanks to my supervisor Professor Dr. José Roberto Castilho Piqueira; you have been a mentor to me. I would like to thank you for encouraging my research and for allowing me to grow as a research scientist. Your advice on both research as well as on my career have been priceless. I would also like to thank Professor Dr. Claudia Wulff from the Department of Mathematics of The University of Surrey who introduced me to the amazing world of symmetries. I thank the University of Surrey for hosting me during part of my research. I also thank UGPN, CNPq, and CAPES for their support. 

We love life, not because we are used to living but because we are used to loving.

(Friedrich Nietzsche) 



\section{Resumo}

Nos últimos anos, tem havido um crescente interesse em estudar redes de osciladores acopladas com retardo de tempo uma vez que estes ocorrem em muitas aplicações da vida real. Em muitos casos, simetria e padrões podem surgir nessas redes; em consequência, uma parte do sistema pode repetir-se, e as propriedades deste subsistema simétrico representam a dinâmica da rede toda. Nesta tese é feita uma análise de uma rede de $N$ nós de segunda ordem totalmente conectada com atraso de tempo. Este estudo é realizado utilizando grupos de simetria. É mostrada a existência de múltiplos valores próprios forçados por simetria, bem como a possibilidade de desacoplamento da linearização no equilíbrio, em representações irredutíveis. É também provada a existência de bifurcações de estado estacionário e Hopf em cada representação irredutível. São usados três modelos diferentes para analisar a dinâmica da rede: o modelo de fase completa, o modelo de fase, e o modelo de diferença de fase. É também determinado um conjunto finito de freqüências $\omega$, que pode corresponder a bifurcações de Hopf em cada caso, para valores críticos do atraso. Apesar de restringir a nossa atenção para nós de segunda ordem, os resultados podem ser estendido para redes de ordem superior, desde que o tempo de atraso nas conexões entre nós permanece igual.

Palavras-chave: Simetria. Grupo de Lie. Rede de osciladores. Sistemas com atraso de tempo. Bifurcações. Equações diferencias com atraso. 


\section{Abstract}

In recent years, there has been increasing interest in studying time-delayed coupled networks of oscillators since these occur in many real life applications. In many cases symmetry, patterns can emerge in these networks; as a consequence, a part of the system might repeat itself, and properties of this symmetric subsystem represent the whole dynamics. In this thesis, an analysis of a second order $N$-node time-delay fully connected network is made. This study is carried out using symmetry groups. The existence of multiple eigenvalues forced by symmetry is shown, as well as the possibility of uncoupling the linearization at equilibria, into irreducible representations due to the symmetry. The existence of steady-state and Hopf bifurcations in each irreducible representation is also proved. Three different models are used to analyze the network dynamics, namely, the full-phase, the phase, and the phase-difference model. A finite set of frequencies $\omega$ is also determined, which might correspond to Hopf bifurcations in each case for critical values of the delay. Although we restrict our attention to second order nodes, the results could be extended to higher order networks provided the time-delay in the connections between nodes remains equal.

Keywords: Symmetry. Lie group. Oscillator network. Time-delay system. Bifurcations. Delay differential equations. 


\section{List of Figures}

Figure 3.1 Symmetry-preserving bifurcation curves for the equilibria $x_{1}^{*}=\phi^{ \pm}$ with $K=1$ and $0<\mu<2$. . . . . . . . . . . . . . 26

Figure 3.2 Real part of the rightmost root for the characteristic function $P_{\mathrm{Fix}\left(\mathbf{S}_{N}\right)}$ with $K=2$ and $x_{1}^{*}=\phi^{+}$, for $\mu=\{0.1,0.2,0.4,0.6,0.8\} . . .27$

Figure 3.3 Real part of the rightmost roots for the characteristic function $P_{\text {Fix }\left(\mathbf{S}_{N}\right)}$ with $K=2$ and $x_{1}^{*}=\phi^{+}$, for $\mu=0.9$ using DDE-Biftool. . 27

Figure 3.4 Symmetry-preserving bifurcation curves for the equilibrium $x_{1}^{*}=$ $\phi^{-}$, with $K=1.05$. . . . . . . . . . . . . . . . . . . . . 29

Figure 3.5 Real part of the rightmost root of $P_{\mathrm{Fix}\left(\mathbf{S}_{N}\right)}$ for $x_{1}^{*}=\phi^{-}, \mu=0.3$, and $K=1.05$, using DDE-Biftool. . . . . . . . . . . . . . . . . 30

Figure 3.6 Curves showing conditions for the existence of symmetry-breaking bifurcations with $x_{1}^{*}=\phi^{+}$and $K>1$. . . . . . . . . . . 34

Figure 3.7 $\quad$ Symmetry-preserving bifurcation curves and symmetry-breaking bifurcation curves for both equilibria $x_{1}^{*}=\phi^{ \pm}$with $N=2$ and $K=1$. 36

Figure 3.8 Real part of the rightmost root for the function $P_{j}$, for $N=2$, $K=1$ and $\mu=\{0.05,0.5,1\} . \ldots \ldots \ldots . \ldots . \ldots . \ldots$

Figure 3.9 Symmetry-preserving bifurcation curves and symmetry-breaking bifurcation curves for $\mu_{+}$and for $\mu_{-}$with $N=3$ and $K=1$, for both equilibria $x_{1}^{*}=\phi^{ \pm}$. . . . . . . . . . . . . . 38

Figure 3.10 For the equilibrium $x_{1}^{*}=\phi^{-}$with $N=3$ and $K=1.05$ curves of symmetry-preserving bifurcations and curves of symmetry-breaking bifurcations. . . . . . . . . . . . . . . . . . . 39

Figure 4.1 Curves $K_{+}\left(\mu ; \omega_{M}, n, m\right)$ from (4.16) and $\delta_{+}(\mu, K(\mu) ; m)$ with $\omega_{M}=$ $1, n=1$ for different values of $m$. . . . . . . . . . . . . 46

Figure $4.2 \quad$ Curves $\widehat{\Omega}$ with $\mu=1, K=1$, and $\omega_{M}=1 . \ldots \ldots \ldots$. . . . . . 47 
Figure 4.3 $S_{n}$ maps for curves $\widehat{\Omega}^{(j)}$ related to the characteristic function $P_{\mathrm{Fix}\left(\mathbf{S}_{N}\right)}$; in all cases, $\delta\left(\omega\left(\tau^{*}\right)\right)$ is positive. . . . . . . . . . . . . 48

Figure $4.4 \quad S_{n}$ maps for different curves $\widehat{\Omega}$ related to the characteristic function $P_{j}$; in all cases, $\delta\left(\omega\left(\tau^{*}\right)\right)$ is positive. . . . . . . . . . . 51

Figure A.1 PLL block diagram $\ldots \ldots \ldots \ldots \ldots \ldots$

Figure A.2 2-node network . . . . . . . . . . . . . . . . . . . 66

Figure A.3 PLL with multiple inputs. . . . . . . . . . . . . . . . . . 67 


\section{List of Symbols}

$\mathbb{C} \quad$ The set of complex numbers

$\operatorname{Fix}(H)$ The fixed-point subspace of $H$

$\operatorname{Im}(z)$ The imaginary part of the complex number $z$

$\mathcal{X} \quad$ The Banach space of continuous functions from $[-\tau, 0]$ into $\mathbb{R}^{N}$

$\mathbb{N} \quad$ The set of natural numbers

$\mathbb{R} \quad$ The set of real numbers

$\operatorname{Re}(z)$ The real part of the complex number $z$

$\mathbf{D}_{m} \quad$ The dihedral group of order $2 \mathrm{~m}$

$\mathbf{S}_{m} \quad$ The symmetric group consisting of all permutations on $m$ symbols

$\mathbf{Z}_{m} \quad$ The cyclic group of order $m$

$\mathbb{Z} \quad$ The set of integers numbers

Acronyms

DDE Delay Differential Equation

PLL Phase Locked Loop

RFDE Retarded Functional Differential Equation

VCO Voltage-controlled oscillator 


\section{Contents}

\begin{tabular}{lll}
\hline 1 & Introduction & 1
\end{tabular}

\begin{tabular}{|lll}
\hline 2 & Literature review & 3
\end{tabular}

2.1 Delay differential equations $\ldots \ldots \ldots \ldots$. . . . . . . . . . . . . . 3

2.2 Characterization of spatio-temporal symmetries . . . . . . . . . . . 5

2.2 .1 Symmetry of solutions . . . . . . . . . . . . . . . . . . 7

$2.2 .2 \quad$ Relative Equilibria $\ldots \ldots \ldots \ldots$

2.3 Method of steps for solution of DDE $\ldots \ldots \ldots \ldots$

2.4 Lambert $W$ function $\ldots \ldots \ldots \ldots$

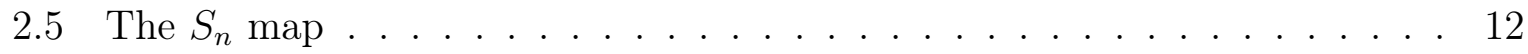

\begin{tabular}{lll}
\hline 3 & Full-phase model & 15
\end{tabular}

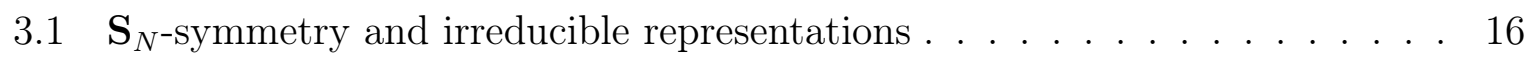

3.2 Symmetry-preserving bifurcations . . . . . . . . . . . . . . . . 21

3.2.1 Roots in the characteristic function $P_{\mathrm{Fix}\left(\mathbf{S}_{N}\right)}$ at $\tau=0$ and as $\tau \rightarrow \infty \quad 22$

3.2 .2 Conditions for the existence of symmetry-preserving bifurcations . . 22

$3.2 .3 \quad$ Curves of symmetry-preserving bifurcations . . . . . . . . . . . 24

3.3 Symmetry-breaking bifurcations . . . . . . . . . . . . . . . . . 30

3.3.1 Roots in the characteristic function $P_{j}$ at $\tau=0$ and as $\tau \rightarrow \infty$. . . 30

3.3.2 Conditions for the existence of symmetry-breaking bifurcations . . . 31

3.3 .3 Curves of symmetry-breaking bifurcations . . . . . . . . . . . 36

3.4 Equivariant Hopf bifurcations for $N=2,3 \ldots \ldots \ldots$. . . . . . . 40

\begin{tabular}{lll}
\hline 4 & Phase model & 41
\end{tabular}

$4.1 \quad$ Symmetry-preserving bifurcations . . . . . . . . . . . . . . . . . . . . . . . 43

4.1 .1 Symmetry-preserving bifurcations from equilibria . . . . . . . . . . 45

$4.1 .2 \quad$ Symmetry-preserving bifurcations from relative equilibria . . . . . . 46 
4.2 Symmetry-breaking bifurcations $\ldots \ldots \ldots \ldots \ldots \ldots$

4.2 .1 Symmetry-breaking bifurcations of relative equilibria . . . . . . . 49

4.2 .2 Symmetry-breaking bifurcations from equilibria $\ldots \ldots \ldots \ldots$

4.2 .3 Curves of symmetry-breaking bifurcations $\ldots \ldots \ldots \ldots$

5 Phase-difference model $\quad 53$

6 Conclusions and Future work 57

6.1 Conclusions . . . . . . . . . . . . . . . . . 57

6.2 Future Work . . . . . . . . . . . . . . . . . . . . 58

\begin{tabular}{|l|l|}
\hline Appendix A The PLL network model & 65
\end{tabular} 


\section{Chapter 1}

\section{Introduction}

Networks of oscillators have been a topic of study for decades since their models can represent several complex dynamics, and results can be applicable in many different areas, such as biology, neurology, economics, and astronomy (Györi and Ladas, 1991; Li, Yang, Wen, and Wang, 2014; Martins and Monteiro, 2013; Piqueira, 2011; Smith, 2011; Strogatz, 2001). Nevertheless, in recent years, much attention has been focused in some emergent behaviors which cannot be explained considering the dynamic of individual nodes isolatedly; those behaviors seem to originate in the network structure itself. In that sense, great effort has been applied to understand the influence that changes in the parameter space associated to the network structure can have over the global dynamics. In this work, we study the influence of a non-ideal connection between nodes in a fully-connected $N$-oscillator network, represented by a constant time-delay. It has already been shown that this delay is responsible for instability, chaotic behavior and bifurcations in oscillators networks; The global exponential stability is explored in Li et al. (2014); Yuan and Zhao (2012), the nonlinear case is studied in Vuellings, Schoell, and Lindner (2014), the linear coupling case is studied in Giannakopoulos and Zapp (2001), and the linear stability for nonlinear coupling is addressed in Olien and Belair (1997); Song and Xu (2013). In our approach, we shall analyze the influence of this time-delay in the emergence of bifurcations from equilibria.

The structure of this thesis is as follows: In chapter two, a literature review is made covering the main concepts and tools used along the thesis, with special emphasis in some useful results related to DDEs; most of the proofs are omitted since we focus on the results and applicability of the concepts. In chapter three, the Full-phase model is introduced 
and a bifurcation analysis is made considering the network symmetry; in this chapter, the main results are presented. In chapters four and five, a comparison between the main results from chapter three and results for the classical Phase model and Phase-difference model are addressed. The conclusions and insights for future research are presented in chapter six. 


\section{Chapter 2}

\section{Literature review}

One of the main issues when dealing with DDEs is the number of roots emerging in the characteristic equation, whilst in ODEs this number is always finite and matches the order of the equation, in DDEs the number of roots is infinite. However, most of the well know results concerning stability and linearization in ODEs can be extended, no without some work, to DDEs. In what follows some important results about retarded differential equations and symmetry, will be presented.

\subsection{Delay differential equations}

Delay differential equations (DDE) which are the class of differential equations that emerge when time-delay is considered in models of oscillators networks are a special case of the socalled Functional differential equations (FDE). In this section, we will review some basics related to FDE applied to DDE following Gilsinn (2009); Gorecki, Fuksa, Grabowski, and Kotytowski (1989); Hale (1977); Krawcewicz and Wu (1999); Kuang (1993); Michiels and Niculescu (2007); Smith (2011); Wu (1998). Let $\tau \geq 0$ be a given constant, $N$ a positive integer and $\mathcal{X}:=\mathcal{C}\left([-\tau, 0], \mathbb{R}^{N}\right)$ the Banach space of continuous functions from $[-\tau, 0]$ into $\mathbb{R}^{N}$ equipped with the usual supremum norm.

$$
\|\varphi\|=\sup _{-\tau \leq \theta \leq 0}|\varphi(\theta)|, \quad \varphi \in \mathcal{X} .
$$

If $x:[-\tau, A] \rightarrow \mathbb{R}^{N}$ is a continuous function with $A>0$ and if $t \in[0, A]$, then $x_{t} \in \mathcal{X}$ is defined by

$$
x_{t}(\theta)=x(t+\theta), \quad \theta \in[-\tau, 0] .
$$


Let the autonomous nonlinear DDE

$$
\dot{x}(t)=f(x(t), x(t-\tau), \eta),
$$

where $\eta \in \mathbb{R}^{p}$ is a vector of parameters, $x(t) \in \mathbb{R}^{N}$, and $x(t-\tau) \in \mathcal{X}$, such that $f\left(x^{*}, x^{*}, \eta\right)=0$, for some equilibrium $x^{*} \in \mathbb{R}^{N}$, clearly $f: \mathbb{R}^{N} \times \mathcal{X} \times \mathbb{R}^{p} \rightarrow \mathbb{R}^{N}$. We can linearize (2.1) around its equilibrium and obtain

$$
\dot{x}(t, \eta)=U(\eta) x(t)+V(\eta) x(t-\tau)+F(x(t), x(t-\tau), \eta)
$$

where $U(\eta)=\frac{\partial}{\partial x(t)} f\left(x^{*}, x^{*}, \eta\right), V(\eta)=\frac{\partial}{\partial x(t-\tau)} f\left(x^{*}, x^{*}, \eta\right)$, and $F(x(t), x(t-\tau), \eta)$ is a nonlinear term. For the linear part of 2.2 the map $T: \mathcal{X} \rightarrow \mathbb{R}^{N}$ is a family of solution operators

$$
(T(t) \phi))(\theta)=\left(x_{t}(\phi)\right)(\theta)=x(t+\theta), \quad \phi \in \mathcal{X}
$$

$x_{t}(\phi)$ is the flow in $\mathcal{X}$ at time $t$. The family of operators $T(t)$ satisfies:

- $T(t)$ is bounded and linear for $t \geq 0$

- $T(0) \phi=\phi$ or $T(0)=I$

- $\lim _{t \rightarrow t_{0}}\left\|T(t) \phi-T\left(t_{0}\right) \phi\right\|=0$

where $\|\cdot\|_{\mathcal{C}}$ is a $\mathcal{X}$ norm. This family of operators is called a semigroup. We can associate to the semigroup $T(t)$ an infinitesimal generator:

$$
A \phi=\lim _{t \rightarrow t_{0}^{+}} \frac{1}{t}[T(t) \phi-\phi]
$$

defined as:

$$
(A(\eta) \phi)=\left\{\begin{array}{cc}
\frac{d \phi}{d \theta} & , \quad-\tau \leq \theta<0 \\
U(\eta) \phi(0)+V(\eta) \phi(-\tau) & , \quad \theta=0,
\end{array}\right.
$$

then $T(t) \phi$ satisfies

$$
\frac{d}{d t} T(t) \phi=A(\eta) T(t) \phi
$$

where $\frac{d}{d t} T(t) \phi=\lim _{h \rightarrow 0} \frac{1}{h}[T(t-h) \phi-T(t) \phi]$. Then the operator form for equation 2.2 is

$$
\frac{d}{d t} x(t ; \phi)=A(\eta) x(t ; \phi)+G(x(t ; \phi), \eta)
$$


$A(\eta)$ and $G(\cdot, \eta)$ map into $\mathcal{X}$, and

$$
(G(\phi ; \eta))(\eta)=\left\{\begin{array}{cc}
0 & , \quad-\tau \leq \theta \leq 0 \\
F(\phi(0), \phi(-\tau), \eta) & ,
\end{array}\right.
$$

The characteristic equation for the linear part of 2.2 is obtained by looking for nontrivial solution of the form $e^{\lambda t} c$ where $c$ is a constant vector. Then the linear part of 2.2 has a nontrivial solution $e^{\lambda t} c$ if and only if

$$
\operatorname{det}(\triangle(\lambda, \tau, \eta))=0
$$

where

$$
\triangle(\lambda, \tau, \eta):=\lambda \mathrm{Id}-L(\eta, \tau)
$$

is the characteristic matrix and the linear operator

$$
L(\eta, \tau):=U(\eta)+V(\eta) e^{-\lambda \tau}
$$

maps $\mathcal{X}$ into $\mathbb{R}^{N}$. We define the transcendental characteristic function associated to the linear part of $(2.2)$ as

$$
P(\lambda, \tau, \eta):=\operatorname{det}(\triangle(\lambda, \tau, \eta))=0
$$

The spectrum $\sigma(A(\eta))$ of $A(\eta)$ consists of eigenvalues which are solutions of (2.4).

\subsection{Characterization of spatio-temporal symmetries}

The oscillator networks studied here present symmetries, which are specified in terms of a group of transformations of the variables in their models that in some sense preserve the structure of the equations and their solutions Collera, 2012, Dias and Rodrigues, 2009, Golubitsky and Stewart, 2002, Golubitsky, Stewart, and Schaeffer, 1988). We shall consider the main following groups:

- $\mathbf{D}_{m}$ the dihedral group of order $2 m$ (rotation and reflections in the plane).

- $\mathbf{Z}_{m}$ the cyclic group of order $m$ (rotations only).

- $\mathbf{S}_{m}$ the symmetric group consisting of all permutations on $m$ symbols; order $m$ !. 
- $\mathbb{R}$ the translational group, which we take to act as

$$
\left(\begin{array}{c}
x_{1} \\
\vdots \\
x_{n}
\end{array}\right) \rightarrow\left(\begin{array}{c}
x_{1} \\
\vdots \\
x_{n}
\end{array}\right)+\left(\begin{array}{c}
C \\
\vdots \\
C
\end{array}\right), \quad C \in \mathbb{R}
$$

Since $f: \mathcal{X} \rightarrow \mathbb{R}^{N}$ in equation 2.1 is a continuously differentiable function with symmetry, then, there exists a compact Lie group $\Gamma$ as well as an orthogonal representation $\rho: \Gamma \rightarrow G L\left(\mathbb{R}^{N}\right)$. For details, see Collera (2012); Ruan, Krawcewicz, Farzamirad, and Balanov (2006).

We say that system 2.1 is equivariant with respect to the action of $\Gamma$ on $\mathbb{R}^{N}$, or $\Gamma$-equivariant, if:

$$
f(\rho(\gamma) \varphi)=\rho(\gamma) f(\varphi), \quad \varphi \in \mathcal{X}, \gamma \in \Gamma
$$

where $\rho(\gamma) \varphi \in \mathcal{C}[-\tau, 0]$ is defined by

$$
(\rho(\gamma) \varphi)(\theta)=\rho(\gamma) \varphi(\theta), \quad \theta \in[-\tau, 0]
$$

The subset $\mathrm{H}$ of $\Gamma$ that fixes a point $\varphi \in \mathcal{X}$ forms a subgroup of $\Gamma$. This subgroup is called the isotropy group of $\varphi$, and is denoted by $H_{\varphi}=\{g \in \Gamma \mid g \varphi=\varphi\}$.

Definition 2.1 (The fixed-point subspace). Let $H$ be a subgroup of $\Gamma$. The fixed-point subspace of $\mathrm{H}$ is given by

$$
\operatorname{Fix}(H)=\{\varphi \in \mathcal{X} \mid h \varphi=\varphi, \forall h \in H\}
$$

If $f$ is $\Gamma$-equivariant and $\mathrm{H}$ is a subgroup of $\Gamma$, then $f(\operatorname{Fix}(H)) \subseteq \operatorname{Fix}(H)$. This means that we can find an equilibrium solution with isotropy subgroup $\mathrm{H}$ by restricting the system in 2.1 to a subspace $\operatorname{Fix}(H)$. In addition, the operator $\triangle(\lambda, \tau, \eta)$ defined in 2.5 is also $\Gamma$-equivariant.

In general, a space $V$ is called $\Gamma$-invariant if $g V \subseteq V$, for all $g \in \Gamma$; as a consequence, it is always possible decompose the space into $\Gamma$-invariants subspaces of smaller dimension. The smallest blocks for such decomposition are said to be irreducible. A space $V$ is $\Gamma$ irreducible if the only $\Gamma$-invariant subspaces of $V$ are $\{0\}$ and $V$ itself. Let $W \subset V$ be a $\Gamma$-invariant subspace. Then there exists a $\Gamma$-invariant complementary subspace $W^{\perp}$ such that

$$
V=W \oplus W^{\perp}
$$


Let $\Gamma$ be a compact Lie group acting on $V$. Then there exists $\Gamma$-irreducible subspaces $V_{1}, \ldots, V_{s}$ of $V$ such that

$$
V=V_{1} \oplus \cdots \oplus V_{s}
$$

this decomposition is not unique and is called Complete Reducibility. Up to $\Gamma$-isomorphism there are a finite number of distinct $\Gamma$-irreducible subspaces of $V$, called $U_{1}, \ldots, U_{t}$. Define $W_{k}$ to be the sum of all $\Gamma$-irreducible subspaces $W$ of $V$ such that $W$ is $\Gamma$-isomorphic to $U_{k}$. Then

$$
V=W_{1} \oplus \cdots \oplus W_{t}
$$

This generates a unique decomposition of $V$ into the so-called isotypic components.

A representation of a group $\Gamma$ on a vector space $V$ is said to be absolutely irreducible if the only linear mapping on $V$ that commutes with $\Gamma$ are scalar multiples of the identity, that is $\{c I \mid c \in \mathbb{R}\}$ is the only mapping that commutes with $\Gamma$.

Therefore, for the dynamics 2.1 of a $N$-oscillator fully connected network with symmetry, for all $N$, there is an $N$-dimensional representation of the symmetric group of order $N$, called the permutation representation, which consists of permuting $N$ coordinates. This has the trivial subrepresentation consisting of vectors whose coordinates are all equal. The orthogonal complement consists of those vectors whose coordinates sum is zero, and when $N \geq 2$, the representation on this subspace is an $N$-1-dimensional irreducible representation, called the standard representation. In other words, $\mathbb{R}^{N}$ decomposes as

$$
\mathbb{R}^{N}=\operatorname{Fix}(H) \oplus U
$$

and $U=\operatorname{Fix}(H)^{\perp} \cong \mathbb{R}^{N-1}$ is $\mathbf{S}_{N^{-}}$invariant and irreducible.

\subsubsection{Symmetry of solutions}

Assume (2.1) has a periodic solution $x(t)$. There are two types of symmetry that make the solution invariant. The first one is the group of spatial symmetries

$$
K=\{\gamma \in \Gamma, \gamma x(t)=x(t), \text { for all } t\}
$$


which is the isotropy group of each point on the solution. The second is the group of spatio-temporal symmetries

$$
H=\left\{\gamma \in \Gamma, \gamma x(t)=x\left(t+t_{0}(\gamma)\right), \text { for all } t\right\}
$$

where $t_{0}(\gamma) \geq 0$. Clearly, spatial symmetries are those spatio-temporal symmetries $\gamma$ for which $t_{0}=0$.

Lemma 2.1. Group $K$ is a normal subgroup of $H$ (Collera, 2012).

Each element $\gamma \in H$ corresponds to a time phase-shift $t_{0}(\gamma)$. If the periodic solution has period $T$, then $t_{0}(\gamma) \in \mathbb{R} / T \mathbb{Z} \cong \mathbf{S}^{1}$. Then the mapping

$$
t_{0}: H \rightarrow \mathbf{S}^{1}
$$

is a homomorphism. Moreover, $\operatorname{ker}\left(t_{0}\right)$ is composed of all $\gamma \in H$ with $t_{0}(\gamma)=0$, that is, $\operatorname{ker}\left(t_{0}\right)=K$. From the first Isomorphism Theorem, given the group homomorphism $t_{0}: H \rightarrow \mathbf{S}^{1}$, we have

$$
\operatorname{Im}\left(t_{0}\right) \cong H / K
$$

which means that $H / K$ is isomorphic to a closed subgroup of $\mathbf{S}^{1}$. Since the only closed subgroups of $\mathbf{S}^{1}$ are $\mathbf{Z}_{m}, m \geq 1$, and itself, we have

$$
H / K \cong \mathbf{S}^{1} \text { or } H / K \cong \mathbf{Z}_{m}, \quad m \geq 1
$$

When $H / K \cong \mathbf{S}^{1}$, the periodic solution is called a rotating wave, while if $H / K \cong \mathbf{Z}_{m}$, the periodic solution is called a discrete rotating wave (Golubitsky et al., 1988).

Theorem 2.1. Let $\Gamma$ be a finite group acting on $\mathcal{X}$. There is a $T$-periodic solution of the $\Gamma$-equivariant system in (2.1) with spatial symmetry $K$ and spatio-temporal symmetry $H$ with

$$
h x(t)=x\left(t-\frac{T}{m}\right)
$$

for some fixed $h \in N(K)$ if and only if

(a) $H / K \cong \mathbf{Z}_{m}$ is cyclic, $m \geq 2$, and $h \in H$ projects onto a generator of $H / K$,

(b) $K$ is an isotropy subgroup, 
(c) $\operatorname{dim} \operatorname{Fix}(K) \geq 2$. If $\operatorname{dim} \operatorname{Fix}(K)=2$, then $H=N(K)$ and $h$ acts on $\operatorname{Fix}(K)$ by rotation through $\pm \frac{2 \pi}{m}$,

(d) $H$ fixes a connected component of $\operatorname{Fix}(K) \backslash L_{K}$, where

$$
L_{K}=\bigcup_{\gamma \neq K} \operatorname{Fix}(\gamma) \cap \operatorname{Fix}(K)
$$

\subsubsection{Relative Equilibria}

An equilibrium point is a point in the phase space that is invariant under the dynamics: $\varphi \in \mathcal{X}$ for which $f(\varphi)=0$ or equivalent $\dot{\varphi}=0$. We can define relative equilibrium as a group orbit that is invariant under the dynamics.

Definition 2.2 (Relative Equilibria). A relative equilibrium is a trajectory $\varphi(t) \in \mathcal{C}[-\tau, 0]$ such that for each $t \in \mathbb{R}$ there is a 1-parameter family of symmetry transformation $\gamma_{t} \in \Gamma$ for which $\varphi(t)=\gamma_{t} \varphi(0)$.

This means the trajectory is contained in a single group orbit. Clearly, if a group orbit is invariant under dynamics, then all the trajectories in it are relative equilibria; conversely, if $\varphi(t)$ is the trajectory through $p$, then $\gamma \varphi(t)$ is the trajectory through $\gamma p$ and accordingly the entire group orbit is invariant (Fiedler, Sandstede, Scheel, and Wulff, 1996, Montaldi, 2000).

Definition 2.3 (Relative periodic orbit). A relative periodic orbit (RPO) of a $\Gamma$-equivariant dynamical system $\dot{X}=F(X)$ with phase space $\mathcal{X}$ is periodic orbit in the space of group orbits $\mathcal{X} / \Gamma$. If $\Gamma$ is compact, then a trajectory $X(t)$ lies on an RPO if and only if it is a periodic orbit in a comoving frame, see, e.g., Fiedler et al. (1996).

\subsection{Method of steps for solution of time-delay differen- tial equations}

Also called sequential integration method, it is the basic form to solve time-delay differential equations with constant delays. The solution is found for each successive interval, one by one, by solving a simple ODE at each interval. Consider the following nonlinear DDE with unique time-delay:

$$
\dot{x}(t)=f(t, x(t), x(t-\tau))
$$


with $\tau>0$. Suppose $f(t, x, y)$ and $\frac{\partial}{\partial x} f(t, x, y)$ are both continuous into $\mathbb{R}^{3}$, let $s \in \mathbb{R}$ and $\phi:[s-\tau, s] \rightarrow \mathbb{R}$ continuous. We are looking for a solution $x(t)$ of $(2.8)$ such that

$$
x(t)=\phi(t), \quad s-\tau \leq t \leq s
$$

and satisfying (2.8) within interval $s \leq t \leq s+\sigma$ for some $\sigma>0$. For $s \leq t \leq s+\tau, x(t)$ has to satisfy the ODE initial condition problem:

$$
\begin{aligned}
& \dot{y}(t)=f(t, y(t), \phi(t-\tau)), \\
& y(s)=\phi(s), \quad s \leq t \leq s+\tau
\end{aligned}
$$

since $f(t, y, \phi(t-\tau))$ and $\frac{\partial}{\partial y} f(t, y)$ are both continuous, thecc solution is guaranteed within interval $s \leq t \leq s+\tau$; then, solution $x(t)$ is defined within interval $[s-\tau, s+\tau]$ and it is possible to extend such solution to the right by repeating the process. In fact, for $s+\tau \leq t \leq s+2 \tau$, solution $x(t)$ of $(2.8)$ and 2.9$)$ have to satisfy the ODE initial condition problem:

$$
\begin{aligned}
\dot{y}(t) & =f(t, y(t), x(t-\tau)), \\
y(s+\tau) & =x(s+\tau), \quad s+\tau \leq t \leq s+2 \tau
\end{aligned}
$$

Theorem 2.2. Let $f(t, x, y)$ and $\frac{\partial}{\partial x} f(t, x, y)$ continuous into $\mathbb{R}^{3}, s \in \mathbb{R}$ and let $\phi$ : $[s-\tau, s] \rightarrow \mathbb{R}$ continuous. Then, some $\sigma>s$ exists and a unique initial condition problem solution 2.8)-2.9 within interval $[s-\tau, \sigma]$.

\subsection{Lambert $W$ function}

Although the number of roots in the characteristic function associated to the linear part in 2.2 is infinite, the stability of the delay equation (2.1) depends on whether there is any root with positive real part or not. Wang (2008) presents a numerical method to compute the rightmost characteristic root for a time-delay linear system using the Lambert $W$ function; the critical poles closest to the imaginary axis, which determine the stability of the system, correspond to the principal branch $\left(W_{0}\right)$ of the Lambert $W$ function. These roots are computed by using Newton-Raphson's and Halley's accelerating schemes (see Mathews and Fink, 2004). The Lambert $W$ function is defined in Asl and Ulsoy (2003) and Corless, Gonnet, Hare, Jeffrey, and Knuth (1996) as the multivalued inverse of the 
function $w \mapsto w e^{w}$, which is the solution of the transcendental equation

$$
w e^{w}=z, \quad z \in \mathbb{C},
$$

solution $W(z)$ has infinite many branches, $W_{k}(z), k \in \mathbb{Z}$, the branches are

$$
\begin{aligned}
& W_{0}(z)=\sum_{n=1}^{\infty} \frac{(-n)^{n-1}}{n !} z^{n}, \\
& W_{k}(z)=\ln _{k}(z)-\ln \left(\ln _{k}(z)\right)+\sum_{l=0}^{\infty} \sum_{m=1}^{\infty} C_{l m} \frac{\left(\ln \left(\ln _{k}(z)\right)\right)^{m}}{\left(\ln _{k}(z)\right)^{l+m}}
\end{aligned}
$$

where $\ln _{k}(z)=\ln (z)+2 \pi k$ i indicates the $k$-th logarithm branch, and the coefficients $C_{l m}$ can be expressed in as $C_{l m}=\frac{(-1)^{l}}{m !}\left[\begin{array}{l}l+m \\ l+1\end{array}\right]$. Shinozaki and Mori (2006) demonstrated that

$$
\operatorname{Re}\left[W_{0}(z)\right]=\max _{k \in \mathbb{Z}} \operatorname{Re}\left[W_{k}(z)\right]
$$

For the time-delay scalar linear system,

$$
\dot{y}(t)+a y(t-\tau)+b y(t)=0,
$$

where $a, b \in \mathbb{C}$ and $y(t) \in \mathbb{R}$, and $\tau>0$. The characteristic equation

$$
\lambda+a e^{-\lambda \tau}+b=0
$$

can be written as

$$
(\lambda+b) \tau e^{(\lambda+b) \tau}=-a \tau e^{b \tau}
$$

and in terms of the Lambert $W$ function, we see that

$$
(\lambda+b) \tau=W_{k}\left(-a \tau e^{b \tau}\right)
$$

and the characteristic roots are

$$
\lambda=\frac{1}{\tau} W_{k}\left(-a \tau e^{b \tau}\right)-b,
$$

which can be computed right away. For the characteristic function

$$
P(\lambda)=\lambda^{2}+a \lambda+b+c e^{-\lambda \tau}=0, \quad a, b, c \in \mathbb{C},
$$

we can compute the rightmost root rewriting it in the form

$$
(a \lambda+b) e^{a \lambda+b}=(a \lambda+b-P(\lambda)) e^{a \lambda+b},
$$


which, expressed in terms of the Lambert $W$ function becomes

$$
a \lambda+b=W_{k}\left((a \lambda+b-P(\lambda)) e^{a \lambda+b}\right),
$$

defining function $G$ for the rightmost root as

$$
G(\lambda):=a \lambda+b-W_{0}\left((a \lambda+b-P(\lambda)) e^{a \lambda+b}\right),
$$

and with a $\lambda_{0}$ be an initial guess, it is possible to calculate the rightmost root of $P(\lambda)$, with these two approaches:

- Newton-Raphson's scheme

$$
\lambda_{i+1}=\lambda_{i}-\frac{G\left(\lambda_{i}\right)}{G^{\prime}\left(\lambda_{i}\right)}, \quad i=0,1,2, \ldots
$$

- Halley's accelerating scheme

$$
\lambda_{i+1}=\lambda_{i}-\frac{G\left(\lambda_{i}\right)}{G^{\prime}\left(\lambda_{i}\right)}\left(1-\frac{G\left(\lambda_{i}\right) G^{\prime \prime}\left(\lambda_{i}\right)}{2\left(G^{\prime}\left(\lambda_{i}\right)\right)^{2}}\right)^{-1}, \quad i=0,1,2, \ldots
$$

where

$$
W_{0}^{\prime}(z)=\frac{W_{0}(z)}{z+z W_{0}(z)}
$$

The success of these two algorithms in finding the rightmost characteristic root strongly depends on how the initial $\lambda_{0}$ guess is chosen, in Wang (2008), and Wang and $\mathrm{Hu}(\sqrt[2008)]{ }$ an auxiliary polynomial is proposed to find the initial $\lambda_{0}$, as the rightmost root for a freely chosen $\hat{\lambda}$

$$
\lambda^{2}+a \lambda+b+c e^{-\hat{\lambda} \tau}=0
$$

clearly, when $\tau=0$, the rightmost root in 2.11 matches with the rightmost root in 2.10 .

\subsection{The $S_{n}$ map}

In order to find bifurcations in 2.1) using the time-delay as a parameter, we will use the method proposed by Beretta and Yang (2002) to find critical imaginary roots for a transcendental function of the form

$$
P(\lambda, \tau)=R(\lambda, \tau)+S(\lambda, \tau) e^{-\lambda \tau}=0
$$


where

$$
R(\lambda, \tau)=\sum_{k=0}^{n} r_{k}(\tau) \lambda^{k}, \quad S(\lambda, \tau)=\sum_{k=0}^{m} s_{k}(\tau) \lambda^{k},
$$

$n, m \in \mathbb{N}_{0}, n>m$, and $r_{k}(\cdot), s_{k}(\cdot): \mathbb{R}_{0}^{+} \rightarrow \mathbb{R}$ are continuous and differentiable functions of $\tau$. Here we shall briefly describe the method.

We are looking for solutions $\lambda= \pm \mathrm{i} \omega$ to $P(\lambda, \tau)$ in 2.12 , with $\omega \in \mathbb{R}^{+}$; since roots appear in complex conjugate pairs (or pure reals), we need look only for positive imaginary roots. Substituting $\lambda=\mathrm{i} \omega$ into 2.12 we have

$$
\begin{aligned}
& \sin (\omega \tau)=\frac{R_{I} S_{R}-S_{I} R_{R}}{|S|^{2}} \\
& \cos (\omega \tau)=-\frac{S_{I} R_{I}+S_{R} R_{R}}{|S|^{2}}
\end{aligned}
$$

where $S_{R}, S_{I}, R_{R}$, and $R_{I}$ stand for the real part and the imaginary part of $S(\mathrm{i} \omega, \tau)$ and $R(\mathrm{i} \omega, \tau)$ respectively, and $|S|$ represents the usual norm of $S(\mathrm{i} \omega, \tau)$.

On the other hand, substituting $\lambda= \pm \mathrm{i} \omega$ into equation (2.12) again, we can eliminate the exponential term and define the polynomial of order $2 n$

$$
F(\omega, \tau):=|R(\mathrm{i} \omega, \tau)|^{2}-|S(\mathrm{i} \omega, \tau)|^{2}=0
$$

Definition 2.4 (The function Arctan). Let $x, y \in \mathbb{R}, r \in \mathbb{R}^{+}$, and $\theta \in[0,2 \pi)$ satisfying $x=r \cos \theta$ and $y=r \sin \theta$. We define the function $\operatorname{Arctan}(\cdot): \mathbb{R} \backslash\{0\} \times \mathbb{R} \backslash\{0\} \rightarrow(-\pi, \pi]$, such that $\operatorname{Arctan}(x, y)=\theta$. This function is the extension of the trigonometrical function $\arctan (y / x): \mathbb{R} \rightarrow(-\pi / 2, \pi / 2)$.

Now, given a $\tau \in \mathbb{R}^{+}$we can compute $\omega=\omega(\tau)$ using polynomial $F$ in 2.15. Since $\sin (\omega \tau)$ and $\cos (\omega \tau)$ in equation 2.14) are both functions of $\omega$ and $\tau$, it is possible to calculate the argument $\theta(\tau)$,

$$
\theta(\tau)=\operatorname{Arctan}\left(-\frac{R_{I} S_{R}-S_{I} R_{R}}{S_{I} R_{I}+S_{R} R_{R}}\right)+2 n \pi \quad \text { for } n \in \mathbb{Z}
$$

then we define the map $\tau_{n}: \mathbb{R}^{+} \rightarrow \mathbb{R}$

$$
\tau_{n}(\tau):=\frac{\theta(\tau)+2 n \pi}{\omega(\tau)}, \quad \omega(\tau) \in \mathbb{R}^{+} .
$$

Finally, if $\tau_{n}$ in 2.16 matches with the given $\tau$, then $\tau=\tau^{*}$ is a bifurcation time-delay, and $\omega\left(\tau^{*}\right)$ is the sought imaginary root to equation 2.12 ; this can be formally expressed by the map

$$
S_{n}:=\tau-\tau_{n}(\tau),
$$


whose zeroes are the critical bifurcation time-delay for equation 2.12.

Now, we need to know in which direction the root found above cross the imaginary axis, if it goes from stable to unstable or from unstable to stable in the complex plane. We need to calculate

$$
\delta\left(\omega\left(\tau^{*}\right)\right):=\operatorname{Re}\left(\left.\frac{d \lambda}{d \tau}\right|_{\lambda=\mathrm{i} \omega\left(\tau^{*}\right)}\right)=\operatorname{Re}\left(-\frac{d P}{d \tau}\left(\frac{d P}{d \lambda}\right)_{\lambda=\mathrm{i} \omega\left(\tau^{*}\right)}^{-1}\right)
$$

thus, from the definition of $P(\lambda, \tau)$ in equation 2.12 we have

$$
\left.\frac{d \lambda}{d \tau}\right|_{\lambda=\mathrm{i} \omega\left(\tau^{*}\right)}=\frac{e^{-\mathrm{i} \omega \tau}\left(\mathrm{i} \omega S(\mathrm{i} \omega, \tau)-S_{\tau}^{\prime}(\mathrm{i} \omega, \tau)\right)-R_{\tau}^{\prime}(\mathrm{i} \omega, \tau)}{R_{\lambda}^{\prime}(\mathrm{i} \omega, \tau)+e^{-\mathrm{i} \omega \tau}\left(S_{\lambda}^{\prime}(\mathrm{i} \omega, \tau)-\tau S(\mathrm{i} \omega, \tau)\right)}
$$

where $R_{\lambda}^{\prime}$ means derivative of $R(\lambda, \tau)$ with respect to $\lambda, R_{\tau}^{\prime}$ derivative of $R(\lambda, \tau)$ with respect to $\tau$ and the same for $S(\lambda, \tau)$. Then we have

$$
\delta\left(\omega\left(\tau^{*}\right)\right)=\frac{A C+B D}{C^{2}+D^{2}}
$$

where

$$
\begin{aligned}
& A=\operatorname{Re}\left(e^{-\mathrm{i} \omega \tau}\left(\mathrm{i} \omega S(\mathrm{i} \omega, \tau)-S_{\tau}^{\prime}(\mathrm{i} \omega, \tau)\right)-R_{\tau}^{\prime}(\mathrm{i} \omega, \tau)\right) \\
& B=\operatorname{Im}\left(e^{-\mathrm{i} \omega \tau}\left(\mathrm{i} \omega S(\mathrm{i} \omega, \tau)-S_{\tau}^{\prime}(\mathrm{i} \omega, \tau)\right)-R_{\tau}^{\prime}(\mathrm{i} \omega, \tau)\right) \\
& C=\operatorname{Re}\left(R_{\lambda}^{\prime}(\mathrm{i} \omega, \tau)+e^{-\mathrm{i} \omega \tau}\left(S_{\lambda}^{\prime}(\mathrm{i} \omega, \tau)-\tau S(\mathrm{i} \omega, \tau)\right)\right) \\
& D=\operatorname{Im}\left(R_{\lambda}^{\prime}(\mathrm{i} \omega, \tau)+e^{-\mathrm{i} \omega \tau}\left(S_{\lambda}^{\prime}(\mathrm{i} \omega, \tau)-\tau S(\mathrm{i} \omega, \tau)\right)\right) .
\end{aligned}
$$

If $\delta\left(\omega\left(\tau^{*}\right)\right)>0$ the root crosses from the left to the right (stable to unstable), and if $\delta\left(\omega\left(\tau^{*}\right)\right)<0$ the root crosses in the opposite direction. It is important to note that condition $\delta\left(\omega\left(\tau^{*}\right)\right) \neq 0$, called transversality condition, is necessary for Hopf bifurcation to occur. 


\section{Chapter 3}

\section{Full-phase model}

In the fully connected $N$-node network under study, each node is a second-order PLL oscillator generating a self-sustained sinusoidal signal whose phase varies according to the weighted mean of the phase differences between the local signal and the signals incoming from the other oscillators. Synchronous state is reached when all these phase differences are equal. Corrections in each local phase to achieve synchronization are possible due to a feedback loop inside each PLL (see Best, 2007; Gardner, 2005); The model we will use in this chapter was presented by Piqueira, Oliveira, and Monteiro (2006) in the original vesion, the so-called "double-frequency" term is neglected arguing that its influence is easily suppressed by the local dynamic in each node (see Acebrón, Bonilla, Pérez Vicente, Ritort, and Spigler, 2005, Bueno, Ferreira, and Piqueira, 2010, Correa and Piqueira, 2013, Gardner, 2005, Kudrewicz and Wasowicz, 2007; Monteiro, dos Santos, and Piqueira, 2003, Piqueira et al., 2006, Piqueira, Orsatti, and Monteiro, 2003). In what follows, we will use this model but include the double-frequency term; thus, we have that equation (3.9) in Piqueira et al. (2006) becomes

$$
\begin{aligned}
\ddot{\phi}_{i}(t)+\mu \dot{\phi}_{i}(t)-\mu \omega_{M}= \\
\frac{K \mu}{N-1} \sum_{\substack{j=1 \\
j \neq i}}^{N}\left[\sin \left(\phi_{j}(t-\tau)-\phi_{i}(t)\right)+\sin \left(\phi_{j}(t-\tau)+\phi_{i}(t)\right)\right],
\end{aligned}
$$

$i=1, \ldots, N$. For details about how to obtain this model, see appendix A. The "doublefrequency" term is embedded in the term $\sin \left(\phi_{j}(t-\tau)+\phi_{i}(t)\right)$. This equation models the dynamics for the $i$-th oscillator in the $N$-node network; we call $\phi_{i}(t)$

$$
\phi_{i}(t):=\theta_{i}(t)+\omega_{M} t, \quad i=1, \ldots, N
$$


the full-phase of the $i$-th oscillator where $\theta_{i}(t)$ is the local instantaneous phase which is rectified by the filter output to achieve the phase of the weighted mean of the incoming signals, $\omega_{M}$ represents the local frequency, $K$ and $\mu$ are gains, and $\tau$ is the time-delay between nodes.

Note that (3.1) has equilibria at $\phi_{i}=\phi^{ \pm}$, where

$$
\begin{aligned}
& 2 \phi^{+}=\arcsin \left(-\frac{\omega_{M}}{K}\right)+2 k \pi, \\
& 2 \phi^{-}=\pi-\arcsin \left(-\frac{\omega_{M}}{K}\right)+2 k \pi,
\end{aligned}
$$

if $\omega_{M} / K \leq 1$.

\section{1 $\mathrm{S}_{N}$-symmetry and irreducible representations in the full-phase model}

We now show that (3.1) has $\mathbf{S}_{N}$-symmetry, where $\mathbf{S}_{N}$ is the group of all permutations $\gamma \in \operatorname{Mat}(n)$ of $N$ elements. A differential equation $\dot{X}(t)=F(X(t))$ posed on the phase space $\mathcal{X}$ defined in section 2.1, is equivariant with respect to the action of a Lie group $\Gamma$ on $\mathcal{X}$ if

$$
\gamma F(X)=F(\gamma X) \text { for all } X \in \mathcal{X}, \gamma \in \Gamma
$$

(see Golubitsky and Stewart, 2002, Golubitsky et al., 1988). We write $\phi=\left(\phi_{1}, \ldots, \phi_{N}\right)$, with $\phi_{j} \in \mathcal{C}([-\tau, 0), \mathbb{R}), j=1, \ldots, N$, and let $x=\left(x^{(1)}, \ldots, x^{(N)}\right) \in \mathcal{X}$ where $x^{(i)}=$ $\left(x_{1}^{(i)}, x_{2}^{(i)}\right)$, and $x_{1}^{(i)}=\phi_{i}$ and $x_{2}^{(i)}=\dot{\phi}_{i}, i=1, \ldots, N$. If $x:[-\tau, A] \rightarrow \mathbb{R}^{n}$ is a continuous function with $A>0$ and if $t \in[0, A]$, then $X(t) \in \mathcal{C}\left([-\tau, 0], \mathbb{R}^{n}\right)$ is defined by

$$
X(t)(\theta)=x(t+\theta), \quad \theta \in[-\tau, 0], t \in[0, A] .
$$

Then, (3.1) takes the form

$$
\frac{\mathrm{d}}{\mathrm{d} t} X(t)=F(X(t), \eta)
$$

where

$$
(F(x))(\theta)=\left\{\begin{array}{cc}
\frac{\partial x}{\partial \theta}(\theta) & , \quad-\tau \leq \theta \leq 0 \\
f(x(0), x(-\tau), \eta) & , \quad \theta=0 .
\end{array}\right.
$$


Here, $\eta=\left(\mu, K, \omega_{M}, \tau\right) \in \mathbb{R}^{4}$ is a parameter and $f=\left(f^{(1)}, \ldots, f^{(N)}\right)$ is such that 3.1 can also be rewritten as an autonomous nonlinear DDE

$$
\dot{x}(t)=f(x(t), x(t-\tau), \eta)
$$

i.e., $f^{(i)}=\left(f_{1}^{(i)}, f_{2}^{(i)}\right)$, thus,

$$
\begin{aligned}
f_{1}^{(i)}\left(x(t), x_{\tau}, \eta\right)= & x_{2}^{(i)}, \\
f_{2}^{(i)}\left(x(t), x_{\tau}, \eta\right)= & -\mu x_{2}^{(i)}+\mu \omega_{M} \\
& +\frac{K \mu}{N-1} \sum_{\substack{j=1 \\
j \neq i}}^{N}\left[\sin \left(x_{1, \tau}^{(j)}-x_{1}^{(i)}\right)+\sin \left(x_{1, \tau}^{(j)}+x_{1}^{(i)}\right)\right],
\end{aligned}
$$

where $x_{\tau}=x(t-\tau), x_{1, \tau}^{(i)}=x_{1}^{(i)}(t-\tau)$. Here $\Gamma=\mathbf{S}_{N}$ acts on $\mathcal{X}$ via

$$
(\gamma \phi)_{j}(\theta)=\left(\gamma \phi_{j}\right)(\theta), \quad \theta \in[-\tau, 0]
$$

Note that $\mathbf{S}_{N}$ is generated by the transpositions $\pi_{i j} \in \operatorname{Mat}(N)$, which swap $\phi_{i}$ with $\phi_{j}$. To show that (3.1) has $\mathbf{S}_{N^{-}}$symmetry, it is thus sufficient to prove that $f \circ \pi_{i j}=\pi_{i j} \circ f$ for all $\pi_{i j}$. We compute that

$$
\begin{aligned}
\left(f \circ \pi_{i j}\right)_{2}^{(i)}\left(x, x_{\tau}\right)= & -\mu x_{2}^{(j)}+\mu \omega_{M} \\
& +\frac{K \mu}{N-1} \sum_{\substack{l=1 \\
\ell \neq j}}^{N}\left[\sin \left(x_{1, \tau}^{(\ell)}-x_{1}^{(j)}\right)+\sin \left(x_{1, \tau}^{(\ell)}+x_{1}^{(j)}\right)\right] \\
= & f_{2}^{(j)}\left(x, x_{\tau}\right) \\
= & \left(\pi_{i j} \circ f\right)_{2}^{(i)}\left(x, x_{\tau}\right) ;
\end{aligned}
$$

Since $\pi_{i j}=\pi_{j i}$, this argument also gives $\left(f \circ \pi_{j i}\right)^{(j)}=\left(\pi_{j i} \circ f\right)^{(j)}$, and, since for all $k \neq i, j$ we have $\left(\pi_{i j} \circ f\right)^{(k)}=f^{(k)}=\left(f \circ \pi_{i j}\right)^{(k)}$ we see that $f$ and $\pi_{i j}$ commute for all $i, j$ which proves $\mathbf{S}_{N^{-} \text {symmetry of } 3.1 \text {. }}$.

Due to the $\mathbf{S}_{N^{-}}$-symmetry acting on $\mathbb{R}^{N}$ by permuting coordinates, $\mathbb{R}^{N}$ decomposes as

$$
\mathbb{R}^{N}=\operatorname{Fix}\left(\mathbf{S}_{N}\right) \oplus U
$$

where for any subgroup $H$ of the action of a Lie group $\Gamma$ on $\mathcal{X}$ the fixed-point subspace of $H$ is given by

$$
\operatorname{Fix}_{\mathcal{X}}(H)=\operatorname{Fix}(H)=\{X \in \mathcal{X} \mid h X=X, \forall h \in H\}
$$


and $U=\left(\operatorname{Fix}\left(\mathbf{S}_{N}\right)\right)^{\perp} \cong \mathbb{R}^{N-1}$ is $\mathbf{S}_{N^{-}}$invariant and irreducible. Moreover $\mathbb{R}^{2 N}$, the phase space for $(\phi, \dot{\phi})$, decomposes as $\mathbb{R}^{2 N}=\operatorname{Fix}\left(\mathbf{S}_{N}\right) \oplus W$ where $\operatorname{Fix}\left(\mathbf{S}_{N}\right)=\mathbb{R}^{2}$, and $W=U \oplus U$ are the isotypic components of the $\mathbf{S}_{N^{-}}$action on $\mathbb{R}^{2 N}$.

Note that the linear operator $A$ defined in $(2.3)$ on a vector space $V$ with a Lie group $\Gamma$ acting on $\mathcal{X}$ is $\Gamma$-invariant, i.e. $g A=A g$ for all $g \in \Gamma$, then, $A$ has a block decomposition, more precisely, $A\left(W_{j}\right) \subseteq W_{j}$ for all isotypic components $W_{j}$ of $V$. Moreover, if $W_{j}$ is the isotypic component of an absolutely irreducible representation $U_{j}$ of dimension $n$ then $\left.A\right|_{W_{j}}$ consists of $n$ identical blocks.

The equilibria $x^{ \pm}$with $\left(x_{1}^{(i)}, x_{2}^{(i)}\right)=\left(\phi^{ \pm}, 0\right), i=1, \ldots, N$, from 3.2 are $\mathbf{S}_{N}$-invariant and hence their linearization $A(\eta)=\mathrm{D} F\left(x^{*}, \eta\right)$, see $(2.3)$, with $F$ as in $(3.3)$, is $\mathbf{S}_{N^{-}}$ equivariant, note that $x^{*}=x^{ \pm}$.

Thus $L(\eta, \tau) \in \operatorname{Mat}(2 N)$ defined in (2.6), can be decomposed as

$$
L \cong\left(\begin{array}{cc}
L_{1} & \\
& L_{N-1}
\end{array}\right),
$$

and

$$
L_{N-1}=\left(\begin{array}{cccc}
L_{2} & & & \\
& L_{2} & & \\
& & \ddots & \\
& & & L_{2}
\end{array}\right),
$$

$L_{1}, L_{2} \in \operatorname{Mat}(2)$. Computing $L$ from (3.4) we get:

$$
L x=\left(\begin{array}{c}
x_{2}^{(1)} \\
K \mu\left(-1+\cos \left(2 x_{1}^{*}\right)\right) x_{1}^{(1)}-\mu x_{2}^{(1)}+\frac{K \mu}{N-1}\left(1+\cos \left(2 x_{1}^{*}\right)\right) e^{-\lambda \tau} \sum_{\substack{j=1 \\
j \neq 1}}^{N} x_{1}^{(j)} \\
\vdots \\
x_{2}^{(N)} \\
K \mu\left(-1+\cos \left(2 x_{1}^{*}\right)\right) x_{1}^{(N)}-\mu x_{2}^{(N)}+\frac{K \mu}{N-1}\left(1+\cos \left(2 x_{1}^{*}\right)\right) e^{-\lambda \tau} \sum_{\substack{j=1 \\
j \neq N}}^{N} x_{1}^{(j)}
\end{array}\right)
$$

Hence, the characteristic matrix $\triangle(\lambda, \tau, \eta) \in \operatorname{Mat}(2 N)$, see (2.5), has the form

$$
\triangle(\lambda, \tau, \eta)=\left(\begin{array}{cccc}
m_{\lambda} & m_{b} & \cdots & m_{r} \\
m_{r} & m_{\lambda} & \cdots & m_{r} \\
\vdots & \vdots & \ddots & \vdots \\
m_{r} & m_{r} & \cdots & m_{\lambda}
\end{array}\right)
$$


where the blocks $m_{\lambda}$ and $m_{r} \in \operatorname{Mat}(2)$ are

$$
m_{\lambda}=\left(\begin{array}{cc}
\lambda & -1 \\
q & \lambda+\mu
\end{array}\right), \quad m_{r}=\left(\begin{array}{ll}
0 & 0 \\
r & 0
\end{array}\right),
$$

with

$$
q=-K \mu\left(-1+\cos \left(2 x_{1}^{*}\right)\right), \quad r=-\frac{K \mu}{N-1}\left(1+\cos \left(2 x_{1}^{*}\right)\right) e^{-\lambda \tau} .
$$

Let $\mathbb{Z}_{N}$ be the cyclic group of order $m$ which are generated by the transformation $\zeta$ that sends $\phi_{j}$ to $\phi_{(j+1)} \bmod N, j=0, \ldots, N-1$. Then each (complex) irreducible representation of $\mathbb{Z}_{N}$, such that $\zeta$ acts as $\mathrm{e}^{2 \pi \mathrm{i} j / N}$, appears exactly once in the permutation representation of $\mathbf{S}_{N}$ on $\mathbb{R}^{N}$, hence the $j$-th isotypic component $W_{j}$ of $\mathbb{Z}_{N}$ on $\mathbb{R}^{2 N}$ is two-dimensional and spanned by

$$
W_{j}=\operatorname{span} \frac{1}{\sqrt{N}}\left(\begin{array}{ccccccc}
\lambda_{0 j} & 0 & \lambda_{1 j} & 0 & \ldots & \lambda_{(N-1) j} & 0 \\
& & & & & & \\
0 & \lambda_{0 j} & 0 & \lambda_{1 j} & \ldots & 0 & \lambda_{(N-1) j}
\end{array}\right)^{T},
$$

where $\lambda_{k j}=\lambda_{(k \cdot j)} \bmod N=\mathrm{e}^{\mathrm{i} \pi((k \cdot j) \bmod N) / N}$ and $W_{0}=\operatorname{Fix}\left(\mathbf{S}_{N}\right)$; then the restriction of the characteristic matrix $\triangle(\lambda, \tau, \eta)$ to $W_{j}$ is

$$
\begin{aligned}
& \left.\triangle(\lambda, \tau, \eta)\right|_{W_{j}}={\overline{W_{j}}}^{T} \triangle(\lambda, \tau, \eta) W_{j} \\
& =\frac{1}{N}\left(\bar{\lambda}_{0 j} I_{2}, \ldots, \bar{\lambda}_{(N-1) j} I_{2}\right)\left(\begin{array}{cccc}
m_{\lambda} & m_{r} & \cdots & m_{r} \\
m_{r} & m_{\lambda} & \cdots & m_{r} \\
\vdots & \vdots & \ddots & \vdots \\
m_{r} & m_{r} & \cdots & m_{\lambda}
\end{array}\right)\left(\begin{array}{c}
\lambda_{0 j} I_{2} \\
\vdots \\
\lambda_{(N-1) j} I_{2}
\end{array}\right) \\
& =\frac{1}{N}\left(\bar{\lambda}_{0 j} m_{\lambda}+m_{r} \sum_{\substack{k=0 \\
k \neq 0}}^{N-1} \bar{\lambda}_{k j}, \ldots, \bar{\lambda}_{(N-1) j} m_{\lambda}+m_{r} \sum_{\substack{k=0 \\
k \neq N-1}}^{N-1} \bar{\lambda}_{k j}\right)\left(\begin{array}{c}
\lambda_{0 j} I_{2} \\
\vdots \\
\lambda_{(N-1) j} I_{2}
\end{array}\right) \\
& =\frac{1}{N}\left(\sum_{\ell=0}^{N-1}\left(m_{\lambda} \lambda_{\ell j}+m_{r} \sum_{\substack{k=0 \\
k \neq \ell}}^{N-1} \lambda_{k j}\right) \bar{\lambda}_{\ell j} I_{2}\right)
\end{aligned}
$$




$$
\begin{aligned}
& =\frac{1}{N}\left(\sum_{\ell=0}^{N-1} m_{\lambda} \lambda_{\ell j} \bar{\lambda}_{\ell j}+m_{r} \sum_{\ell=0}^{N-1} \sum_{\substack{k=0 \\
k \neq \ell}}^{N-1} \lambda_{k j} \bar{\lambda}_{\ell j}\right) \\
& =m_{\lambda}+\frac{1}{N} m_{r} \sum_{\ell=0}^{N-1} \sum_{\substack{k=0 \\
k \neq \ell}}^{N-1} \lambda_{k j} \bar{\lambda}_{\ell j} .
\end{aligned}
$$

Moreover,

$$
\begin{aligned}
& \text { if } j=0, \quad \sum_{\ell=0}^{N-1} \sum_{\substack{k=0 \\
k \neq \ell}}^{N-1} \lambda_{k j} \bar{\lambda}_{\ell j}=N(N-1) \\
& \text { if } j \neq 0, \quad \sum_{\ell=0}^{N-1} \sum_{\substack{k=0 \\
k \neq \ell}}^{N-1} \lambda_{k j} \bar{\lambda}_{\ell j}=-\sum_{\ell=0}^{N-1} \lambda_{\ell j} \bar{\lambda}_{\ell j}=-N .
\end{aligned}
$$

Therefore

$$
\left.\triangle(\lambda, \tau, \eta)\right|_{W_{j}}= \begin{cases}m_{\lambda}+(N-1) m_{r}, & j=0, \\ m_{\lambda}-m_{r}, & j=1, \ldots, N-1 .\end{cases}
$$

The characteristic matrix decomposition is

$$
\triangle(\lambda, \tau, \eta)=\operatorname{diag}\left(\left.\triangle\right|_{\operatorname{Fix}\left(\mathbf{S}_{N}\right)},\left.\triangle\right|_{W_{1}}, \ldots,\left.\triangle\right|_{W_{N-1}}\right),
$$

where $\left.\triangle\right|_{\operatorname{Fix}\left(\mathbf{S}_{N}\right)}=\left.\triangle(\lambda, \tau, \eta)\right|_{W_{0}}$.

The characteristic function $P(\lambda, \tau, \eta)$ defined in equation (2.7) becomes

$$
P(\lambda, \tau, \eta)=\operatorname{det}\left(\left.\triangle(\lambda, \tau, \eta)\right|_{\operatorname{Fix}\left(\mathbf{S}_{N}\right)}\right) \prod_{j=1}^{N-1} \operatorname{det}\left(\left.\triangle(\lambda, \tau, \eta)\right|_{W_{j}}\right)=0
$$

or

$$
P(\lambda, \tau, \eta)=\operatorname{det}\left(m_{\lambda}+(N-1) m_{r}\right)\left(\operatorname{det}\left(m_{\lambda}-m_{r}\right)\right)^{N-1}=0,
$$

and using (3.6) we obtain

$$
\begin{aligned}
P_{\mathrm{Fix}\left(\mathbf{S}_{N}\right)}(\lambda, \tau, \eta) & =\operatorname{det}\left(\left.\triangle(\lambda, \tau, \eta)\right|_{\mathrm{Fix}\left(\mathbf{S}_{N}\right)}\right)=0 \\
& =\lambda^{2}+\mu \lambda+q+(N-1) r=0 \\
P_{j}(\lambda, \tau, \eta) & =\operatorname{det}\left(\left.\triangle(\lambda, \tau, \eta)\right|_{W_{j}}\right)=0 \\
& =\lambda^{2}+\mu \lambda+q-r=0,
\end{aligned}
$$


for $j=1, \ldots, N-1$, where $q$ and $r$ are as in (3.7).

From the equilibria in 3.2 we have

$$
\cos \left(2 x_{1}^{*}\right)= \pm \frac{1}{K} \sqrt{K^{2}-\omega_{M}^{2}}, \text { if } x_{1}^{*}=\phi^{ \pm}
$$

with $K \geq \omega_{M}$ in order to keep $\phi_{1,2} \in \mathbb{R}$. By scaling $\widetilde{K}=K / \omega_{M}, \tilde{\mu}=\mu / \omega_{M}, \tilde{\lambda}=\lambda / \omega_{M}$, and $\tilde{\tau}=\omega_{M} \tau$, and removing the tilde in the variables we obtain the normalized equilibria:

$$
\cos \left(2 x_{1}^{*}\right)= \pm \sqrt{1-\frac{1}{K^{2}}}, \text { if } x_{1}^{*}=\phi^{ \pm}
$$

with

$$
K \geq 1
$$

\section{$3.2 \quad$ Symmetry-preserving bifurcations}

In the next two sections, bifurcations in the two isotypic components $\operatorname{Fix}\left(\mathbf{S}_{N}\right)$ and $V$ of $\mathbf{S}_{N}$ found previously will be analyzed, conditions for the existence of eigenvalues $\lambda= \pm \mathrm{i} \omega$ with $\omega \in \mathbb{R}^{+}$shall be given in terms of parameters $K, \mu, \tau \in \mathbb{R}^{+}$, and $N \in \mathbb{N}>1$; and the critical time delays $\tau$ switching stability shall be computed.

When $\tau=0$, the transcendental characteristic functions in 3.9 become ordinary characteristic polynomials with two roots each. Since we are interested in analyzing the influence of the time-delay between the nodes in the network, it is important to know whether the system is stable or not at $\tau=0$. If it is, we would like to determine if there exists some $\tau \in \mathbb{R}^{+}$such that a finite number of roots cross the imaginary axis from the left to the right switching stability with $d \lambda /\left.d \tau\right|_{\lambda=\mathrm{i} \omega} \neq 0$. For this analysis we use the $S_{n}$ map which was discussed in section 2.5. If some roots are unstable at the equilibrium at $\tau=0$, we will look for some $\tau \in \mathbb{R}^{+}$such that all unstable roots (always a finite number) cross from the right to the left at $\tau$, switching stability from unstable to stable; this task will be addressed using the Lambert W function (see Asl and Ulsoy, 2003, Corless et al., 1996; Mathews and Fink, 2004; Wang, 2008).

We will start analyzing bifurcations in $\operatorname{Fix}\left(\mathbf{S}_{N}\right)$. Note that $\mathbf{S}_{N^{-}}$symmetry implies that 3.3 maps $\operatorname{Fix}_{\mathcal{X}}\left(\mathbf{S}_{N}\right)$, which means that we can restrict 3.1 to subspace $\operatorname{Fix}\left(\mathbf{S}_{N}\right)$. These bifurcations preserve the $\mathbf{S}_{N}$-symmetry, causing bifurcating periodic orbits to be synchronized, i.e., satisfying, $\phi_{i}=\phi_{j}$ for all $i, j$. In section 3.3 we will study bifurcation 
in the other blocks which are symmetry-breaking, i.e., bifurcating periodic orbits are not fully-synchronized.

\subsubsection{Roots in the characteristic function $P_{\mathrm{Fix}\left(\mathrm{S}_{N}\right)}$ at $\tau=0$ and as}

$$
\tau \rightarrow \infty
$$

In equation $(3.9)$, when $\tau=0$, we have two roots

$$
\lambda_{ \pm}=-\frac{1}{2} \mu \pm \frac{1}{2}\left(\mu^{2}+8 K \mu \cos \left(2 x_{1}^{*}\right)\right)^{1 / 2}
$$

substituting the two equilibria $x_{1}^{*}=\phi^{ \pm}$from 3.10 we obtain two cases:

$$
\lambda_{ \pm}=-\frac{1}{2} \mu \pm \frac{1}{2}\left(\mu^{2} \pm 8 \mu \sqrt{K^{2}-1}\right)^{1 / 2}
$$

and having in mind $K \geq 1$, see (3.11), we observe that

- If $K>1$, there is an unstable root for $x_{1}^{*}=\phi^{+}$, and both roots are stable when $x_{1}^{*}=\phi^{-}$.

- If $K=1$, there is a constant root at $\lambda=0$ and another one at $\lambda=-\mu$, for both equilibria $x_{1}^{*}=\phi^{ \pm}$.

In the limit when $\tau \rightarrow \infty$ in equation $(3.9)$ for both equilibria $\phi^{ \pm}$, assuming that $\operatorname{Re}(\lambda)>$ 0 , the roots are:

$$
\lambda_{ \pm}=-\frac{1}{2} \mu \pm \frac{1}{2}\left(\mu^{2}-4 K \mu\left(1 \mp \sqrt{1-\frac{1}{K^{2}}}\right)\right)^{1 / 2}
$$

but these roots are not on the right-side of the complex plane neither for $\phi^{+}$nor $\phi^{-}$, which is a contradiction; therefore, at $\tau \rightarrow \infty$ both equilibria are spectrally stable in $\operatorname{Fix}\left(\mathbf{S}_{N}\right)$.

\subsubsection{Conditions for the existence of symmetry-preserving bifurc- ations}

Necessary but not sufficient conditions for the existence of roots $\lambda= \pm \mathrm{i} \omega, \omega \in \mathbb{R}^{+}$; in $\operatorname{Fix}\left(\mathbf{S}_{N}\right)$ they are given by the polynomial $F(\omega)$ in $(2.15)$. From $(2.12)$, 2.13$)$ with $n=2$ and $m=0$ and $(3.9)$ we have

$$
\begin{aligned}
& R(\lambda, \eta)=\lambda^{2}+\mu \lambda+K \mu\left(1-\cos \left(2 x_{1}^{*}\right)\right) \\
& S(\lambda, \eta)=-K \mu\left(1+\cos \left(2 x_{1}^{*}\right)\right),
\end{aligned}
$$


then $F(\omega)$ becomes

$$
F(\omega)=\omega^{4}+\left(\mu^{2}-2 K \mu\left(1-\cos \left(2 x_{1}^{*}\right)\right)\right) \omega^{2}-4 K^{2} \mu^{2} \cos \left(2 x_{1}^{*}\right)=0
$$

and

$$
\begin{aligned}
\omega_{ \pm}^{2}= & -\frac{1}{2}\left(\mu^{2}-2 K \mu\left(1-\cos \left(2 x_{1}^{*}\right)\right)\right) \\
& \pm \frac{1}{2}\left[\left(\mu^{2}-2 K \mu\left(1-\cos \left(2 x_{1}^{*}\right)\right)\right)^{2}+16 K^{2} \mu^{2} \cos \left(2 x_{1}^{*}\right)\right]^{1 / 2} .
\end{aligned}
$$

For the sake of notation we write

$$
\omega_{ \pm}^{2}=-\frac{1}{2} b \pm \frac{1}{2} \sqrt{b^{2}-4 c}
$$

where

$$
\begin{aligned}
& b=\mu^{2}-2 K \mu\left(1-\cos \left(2 x_{1}^{*}\right)\right) \\
& c=-4 K^{2} \mu^{2} \cos \left(2 x_{1}^{*}\right),
\end{aligned}
$$

with $\cos \left(2 x_{1}^{*}\right)= \pm \sqrt{1-\left(1 / K^{2}\right)}$.

Lemma 3.1. A necessary condition for the existence of $\omega_{ \pm} \in \mathbb{R}$ is

$$
b^{2}-4 c \geq 0
$$

Moreover,

1. If $b \geq 0$

(a) If $c \leq 0$ then $\omega_{+} \in \mathbb{R}_{0}^{+}$and $\omega_{-} \in \mathbb{C} \backslash \mathbb{R}$.

(b) If $c>0$ then $\omega_{ \pm} \in \mathbb{C} \backslash \mathbb{R}$.

2. If $b<0$

(a) If $c \leq 0$ then $\omega_{+} \in \mathbb{R}^{+}, \omega_{-} \in \mathbb{C} \backslash \mathbb{R}$.

(b) If $c>0$ then $\omega_{ \pm} \in \mathbb{R}^{+}$.

Provided $\omega_{+} \in \mathbb{R}$ or $\omega_{-} \in \mathbb{R}$, we can find the critical time-delay $\tau \in \mathbb{R}^{+}$such that $\lambda= \pm \mathrm{i} \omega$ is a root of $P_{\operatorname{Fix}\left(\mathbf{S}_{N}\right)}$ using the $S_{n}$ map introduced in section 2.5; thus, from 2.14 and (2.16), we have

$$
\begin{aligned}
\sin \left(\omega_{ \pm} \tau\right) & =-\frac{\omega_{ \pm}}{K\left(1+\cos \left(2 x_{1}^{*}\right)\right)} \\
\cos \left(\omega_{ \pm} \tau\right) & =\frac{-\omega_{ \pm}^{2}+K \mu\left(1-\cos \left(2 x_{1}^{*}\right)\right)}{K \mu\left(1+\cos \left(2 x_{1}^{*}\right)\right)}
\end{aligned}
$$


and

$$
\tau_{ \pm}\left(\omega_{ \pm} ; K, \mu, n\right)=\frac{1}{\omega_{ \pm}}\left[\operatorname{Arctan}\left(\frac{\sin \left(\omega_{ \pm} \tau\right)}{\cos \left(\omega_{ \pm} \tau\right)}\right)+2 n \pi\right], \quad n \in \mathbb{Z} .
$$

Here we want to stress that $\tau_{ \pm}$does not depend on $\tau$. Next, we will write $\tau_{ \pm}(n)=\tau\left(\omega_{ \pm}, n\right)$ to emphasize the dependence on $\omega_{ \pm}$or $n$, respectively, according to need.

The direction in which the roots cross the imaginary axis, if there are any, can be obtained by looking at the sign of $\delta(\omega)$ defined in 2.19 , where the constants from 2.20 are

$$
\begin{aligned}
A & =\mu \omega_{ \pm}^{2} \\
B & =\omega_{ \pm}^{3}-K \mu \omega_{ \pm}\left(1-\cos \left(2 x_{1}^{*}\right)\right) \\
C & =\mu-\tau_{ \pm} \omega_{ \pm}^{2}+\tau_{ \pm} K \mu\left(1-\cos \left(2 x_{1}^{*}\right)\right) \\
D & =\omega_{ \pm}\left(2+\mu \tau_{ \pm}\right) .
\end{aligned}
$$

It is clear from 2.19 that the sign of $\delta$ depends on the numerator $A C+B D$; thus using (3.21), we compute

$$
A C+B D=\omega_{ \pm}^{2}\left(2 \omega_{ \pm}^{2}+b\right)
$$

but, from equation (3.17), we know that $b=-2 \omega_{ \pm}^{2} \pm \sqrt{b^{2}-4 c}$, then substituting into 3.22 we have

$$
A C+B D= \pm \omega_{ \pm}^{2} \sqrt{b^{2}-4 c}
$$

thus the sign of $\delta\left(\omega_{ \pm}\right)$is

$$
\operatorname{sign}\left(\delta\left(\omega_{ \pm}\right)\right)=\left\{\begin{array}{lll}
+1 & \text { for } & \omega_{+} \\
-1 & \text { for } & \omega_{-}
\end{array}\right.
$$

\subsubsection{Curves of symmetry-preserving bifurcations}

Now, we shall analyze the bifurcation curves in $\operatorname{Fix}\left(\mathbf{S}_{N}\right)$, from which fully synchronized periodic orbits emanate, in three cases:

- When $K=1$ and for both equilibria $\phi^{ \pm}$, roots of the characteristic function $P_{\operatorname{Fix}\left(\mathbf{S}_{N}\right)}$ in 3.9 when $\tau=0$ are,

$$
\lambda_{+}=0, \quad \lambda_{-}=-\mu .
$$


The equation $F(\omega)=0$ in 3.15 , which represents a necessary condition for the existence of roots at $\lambda= \pm \mathrm{i} \omega$, becomes

$$
F(\omega)=\omega^{4}+\left(\mu^{2}-2 \mu\right) \omega^{2}=0
$$

and here we have $\omega=0$ and two other roots

$$
\omega_{ \pm}^{2}=-\frac{1}{2}\left(\mu^{2}-2 \mu\right) \pm \frac{1}{2} \sqrt{\left(\mu^{2}-2 \mu\right)^{2}}
$$

clearly, these conjugate roots exist provided the condition

$$
0<\mu<2
$$

holds true; then we have

$$
\begin{aligned}
& \omega_{-}=0 \\
& \omega_{+}= \pm \sqrt{2 \mu-\mu^{2}}, \quad \omega \in \mathbb{R}^{+} .
\end{aligned}
$$

If condition 3.25 does not hold, roots in $P_{\mathrm{Fix}\left(\mathbf{S}_{N}\right)}$ will remain on the left hand side of the complex plane with a constant zero root, for all $\tau \in \mathbb{R}^{+}$. When $\mu=2$, there are two constant zero roots of $P_{\operatorname{Fix}\left(\mathbf{S}_{N}\right)}$. The other necessary condition to $\omega \in \mathbb{R}$ is related to the $\sin \left(\omega_{+} \tau\right)$ and $\cos \left(\omega_{+} \tau\right)$ functions, thus from 2.14 and 3.14 with $R_{R}=-\omega_{+}^{2}+\mu, R_{I}=\mu \omega_{+}, S_{R}=-\mu$ and $S_{I}=0$ we obtain

$$
\begin{aligned}
\sin \left(\omega_{+} \tau\right) & =-\omega_{+} \\
\cos \left(\omega_{+} \tau\right) & =\frac{\mu-\omega_{+}^{2}}{\mu} .
\end{aligned}
$$

Combining (3.24) and (3.26), we compute $\tau$ as a function of $\omega_{+}$and the other free parameters,

$$
\tau_{+}\left(\omega_{+} ; \mu, n\right)=\frac{1}{\omega_{+}}\left[\operatorname{Arctan}\left(\frac{-\omega_{+} \mu}{-\omega_{+}^{2}+\mu}\right)+2 n \pi\right], \quad n \in \mathbb{Z}
$$

We already know from $(3.23)$ that roots $\lambda= \pm \mathrm{i} \omega_{+}$cross the imaginary axis from the left to the right. In figure 3.1. curves for $\tau\left(\omega_{+} ; \mu, n\right)$ from 3.27) are shown for different values of $n$, only positive values of $\tau$ are considered which correspond to $n \in \mathbb{N}$. Curve $\tau_{+}(n)=\tau\left(\omega_{+} ; \mu, n\right)$ for $n=1$ determines the first root crossing from the left to the right. For each curve $\tau_{+}(n)$ with $n>1$, a new root crosses from the left-hand side to the right-hand side of the imaginary axis. 
Remark. Although the equilibria are spectrally stable in $\operatorname{Fix}\left(\mathbf{S}_{N}\right)$ (no roots of $P_{\operatorname{Fix}\left(\mathbf{S}_{N}\right)}$ with positive real part) with $K=1$ at $\tau=0$ for both equilibria $x_{1}^{*}=\phi^{ \pm}$, see 3.12 , and the first bifurcation root appears on curve $\tau_{+}(\mu ; 1)$, nothing can be said about the stability of the equilibria in $\operatorname{Fix}\left(\mathbf{S}_{N}\right)$ below this curve because there exists a constant zero root $\left(\omega_{-}=0\right)$.

- Next, we analyze the unstable equilibrium $x_{1}^{*}=\phi^{+}$when $K>1$. We are interested in any values of parameters $\mu$ and $\tau$ such that roots in $P_{\mathrm{Fix}\left(\mathbf{S}_{N}\right)}$ become stable, i.e., if in any $\mu, \tau \in \mathbb{R}^{+}$for which we have $\max (\operatorname{Re}(\lambda))<0$, for all roots $\lambda$ of $P_{\operatorname{Fix}\left(\mathbf{S}_{N}\right)}$. We shall use the Lambert W function (see Asl and Ulsoy, 2003; Corless et al., 1996. Mathews and Fink, 2004, Wang, 2008), to find the rightmost root when $\mu$ and $\tau$ vary.

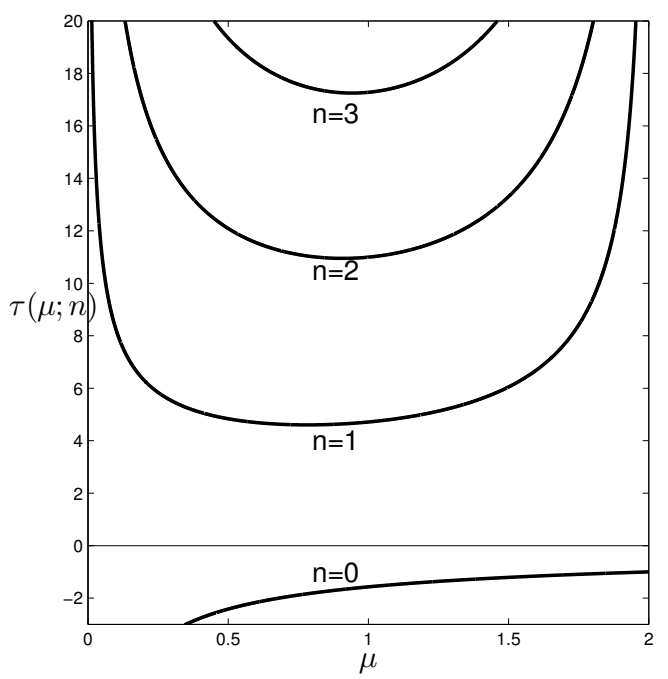

Figure 3.1: Symmetry-preserving bifurcation curves for the equilibria $x_{1}^{*}=\phi^{ \pm}$, with $K=1$ and $0<\mu<2$.

The initial $\lambda_{0}$ guess needed in both Newton's and Halley's schemes used to calculate the rightmost root is found using the rightmost root in an auxiliary polynomial as proposed in Wang and $\mathrm{Hu}$ (2008); Wang (2008), and for the following iterations, the root found in the previous iteration is used as an initial guess. Results of the numerical simulation with $\mu=\{0.1,0.2,0.4,0.6,0.8\}$ and $K=2$ are shown in figure 3.2. As expected at $\tau=0$, the real part of the rightmost root is positive and increases monotonically with $\mu$, see $(3.12)$. On the other hand, when $\tau$ grows the real part of the rightmost root tends to a non-positive value as shown in (3.13) and 
predicted in Section 3.2.1.
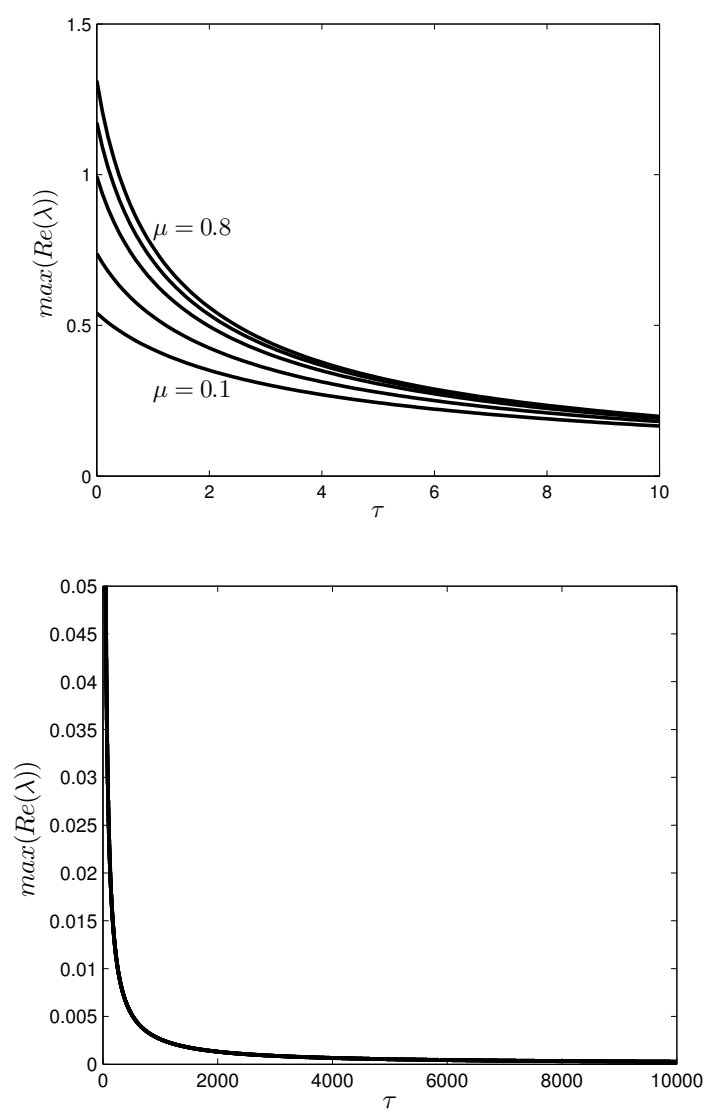

Figure 3.2: Real part of the rightmost root for the characteristic function $P_{\mathrm{Fix}\left(\mathbf{S}_{N}\right)}$ with $K=2$ and $x_{1}^{*}=\phi^{+}$, for $\mu=\{0.1,0.2,0.4,0.6,0.8\}$.

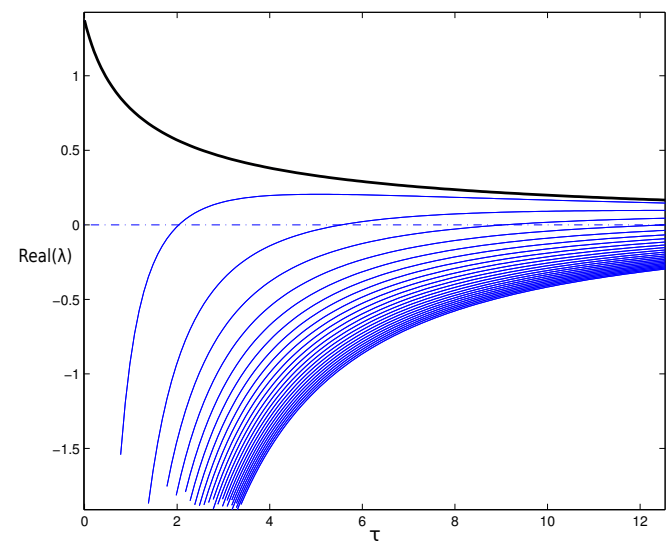

Figure 3.3: Real part of the rightmost roots for the characteristic function $P_{\operatorname{Fix}\left(\mathbf{S}_{N}\right)}$ with $K=2$ and $x_{1}^{*}=\phi^{+}$, for $\mu=0.9$ using DDE-Biftool.

Using the Matlab ${ }^{\circledR}$ routines DDE-Biftool Engelborghs, Luzyanina, and Roose, 2002; Engelborghs, Luzyanina, and Samaey, 2001) we observe that the other char- 
acteristic roots converge when $\tau \rightarrow \infty$, see figure 3.3. Although roots can become stable when $\tau \rightarrow \infty$, we are interested only in finite values of time-delay; consequently, there is numerical evidence that some roots in $P_{\operatorname{Fix}\left(\mathbf{S}_{N}\right)}$ remain unstable when $x_{1}^{*}=\phi^{+}$for any finite value of $\mu, \tau \in \mathbb{R}^{+}$.

- For the equilibrium $x_{1}^{*}=\phi^{-}$with $K>1$, the characteristic function $P_{\operatorname{Fix}\left(\mathbf{S}_{N}\right)}$ in equation 3.9 becomes

$$
P_{\mathrm{Fix}\left(\mathbf{S}_{N}\right)}=\lambda^{2}+\mu \lambda+K \mu\left(1+\sqrt{1-\frac{1}{K^{2}}}\right)-K \mu\left(1-\sqrt{1-\frac{1}{K^{2}}}\right) e^{-\lambda \tau}=0 .
$$

From (3.12), we know both roots are stable when $\tau=0$ and, from (3.13), we also know there are no roots on the right-side of the complex plane when $\tau \rightarrow \infty$.

From $F(\omega)=0$ in 3.15 with $x_{1}^{*}=\phi^{-}$, we obtain

$$
\begin{aligned}
\omega_{ \pm}^{2}= & -\frac{1}{2}\left(\mu^{2}-2 K \mu\left(1+\sqrt{1-\frac{1}{K^{2}}}\right)\right) \\
\pm & \frac{1}{2}\left[\left(\mu^{2}-2 K \mu\left(1+\sqrt{1-\frac{1}{K^{2}}}\right)\right)^{2}-16 K^{2} \mu^{2} \sqrt{1-\frac{1}{K^{2}}}\right]^{1 / 2} .
\end{aligned}
$$

Then, by Lemma 3.1 with $c>0, \omega \in \mathbb{R}^{+}$if and only if the discriminant is positive and $b<0$, i.e., the first term is positive, which implies

$$
\mu<2\left(K+\sqrt{K^{2}-1}\right) .
$$

The discriminant is positive if and only if

$$
\left|\mu-2\left(K+\sqrt{K^{2}-1}\right)\right|>4 \sqrt{K \sqrt{K^{2}-1}}
$$

hence, by 3.28

$$
\mu<2\left(K+\sqrt{K^{2}-1}\right)-4 \sqrt{K \sqrt{K^{2}-1}}=: \mu_{\max }
$$

is a necessary condition for the existence of bifurcations in $\operatorname{Fix}\left(\mathbf{S}_{N}\right)$ when $x_{1}^{*}=\phi^{-}$ with $K>1$. When $K=1$, this condition becomes condition (3.25).

Remark. We know roots in $P_{\mathrm{Fix}\left(\mathbf{S}_{N}\right)}$ are stable when $x_{1}^{*}=\phi^{-}$for $K \geq 1$ at $\tau=0$, see (3.12); using the time-delay as parameter, bifurcations can occur for time delays $\tau$ satisfying (3.20) provided condition (3.29) holds. If condition (3.29) does not hold, then roots of $P_{\mathrm{Fix}\left(\mathbf{S}_{N}\right)}$ will remain stable for all $\tau$. In that sense, $\mu_{\max }$ sets the lower limit to $\mu$ for the stability of the equilibrium in $\operatorname{Fix}\left(\mathbf{S}_{N}\right)$ for all time delays $\tau$ in this case. 


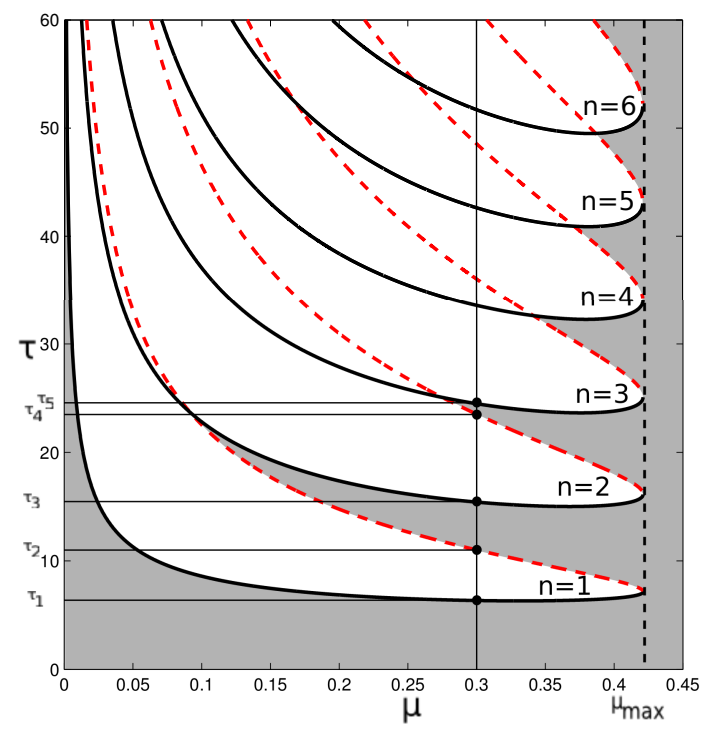

Figure 3.4: Symmetry-preserving bifurcation curves for the equilibrium $x_{1}^{*}=\phi^{-}$, with $K=1.05$. Within the shadowed region, there are no roots with positive real part.

As an example, we choose $K=1.05$; with this value, condition 3.29 becomes $0<\mu<\mu_{\max }=0.4211$. With these parameters and using (3.16), 3.19), 3.20), and (2.19), for $x_{1}^{*}=\phi^{-}$, curves corresponding to $\tau_{ \pm}$are presented in figure 3.4 for different values of $n\left(\tau_{+}\right.$in solid line and $\tau_{-}$in dashed line), a lobe $\tau_{+}(n)$ and $\tau_{-}(n)$ is plotted for each $n$; note that only positive values of $\tau$ are considered. We already know from (3.23) that $\delta_{+}\left(\omega_{+}\right)>0$ and $\delta_{-}\left(\omega_{-}\right)<0$, thus the shadowed area indicates the region where the equilibrium is stable in $\operatorname{Fix}\left(\mathbf{S}_{N}\right)$.

These results are tested using DDE-Biftool (Engelborghs et al., 2002, 2001); with $\mu=0.3$ and $K=1.05$, we found the critical time delays $\tau_{1}$ to $\tau_{5}$ leading to Hopf bifurcations $\left(\tau_{1}=6.34, \tau_{2}=11, \tau_{3}=15.41, \tau_{4}=23.51\right.$, and $\left.\tau_{5}=24.48\right)$ which match with those found using the $S_{n}$ map, see figure 3.4 . In figure 3.5, the real part of the rightmost root is shown as a black curve, and the critical time delays $\tau_{1}$ to $\tau_{5}$ are also shown. Each peak is related to the corresponding lobe in figure 3.4 . the numerics confirms that at $\tau_{1}, \tau_{3}$ and $\tau_{5}$, the rightmost root crosses from the left to the right of the imaginary axis switching stability from stable to unstable and, at $\tau_{2}, \tau_{4}$, the rightmost root come back to the left-hand side of the complex plane, switching stability from unstable to stable again; these time delays are the same as shown in figure 3.4. Clearly for $\tau>\tau_{5}$, the equilibrium becomes unstable 
in $\operatorname{Fix}\left(\mathbf{S}_{N}\right)$. Thus, for the given parameters the equilibrium is stable in $\operatorname{Fix}\left(\mathbf{S}_{N}\right)$ within the interval $\left(0, \tau_{1}\right) \cup\left(\tau_{2}, \tau_{3}\right) \cup\left(\tau_{4}, \tau_{5}\right)$, when $\mu<\mu_{\max }$.

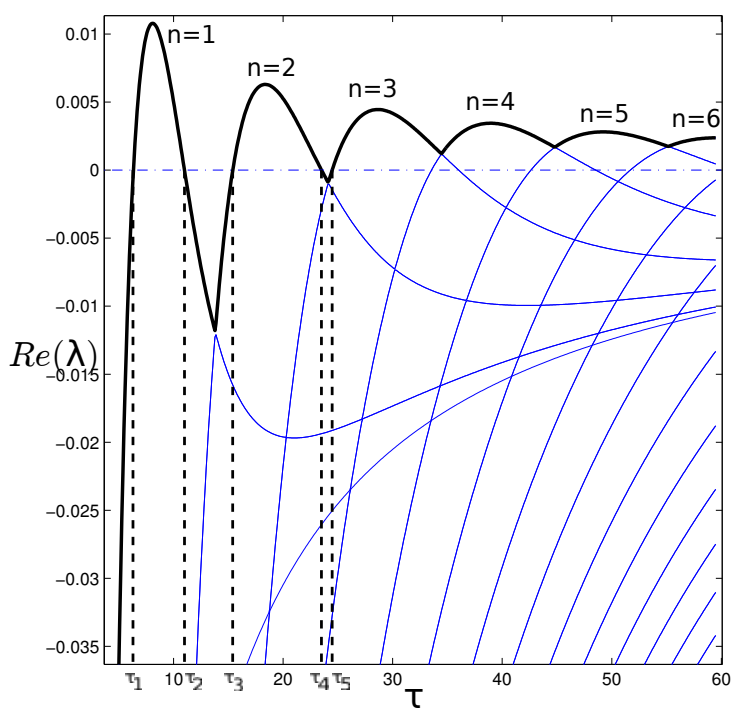

Figure 3.5: Real part of the rightmost root of $P_{\mathrm{Fix}\left(\mathbf{S}_{N}\right)}$ for $x_{1}^{*}=\phi^{-}, \mu=0.3$, and $K=1.05$, using DDE-Biftool.

\subsection{Symmetry-breaking bifurcations}

In this section we shall analyze conditions for existence of bifurcations in $W_{j}$.

\subsubsection{Roots in the characteristic function $P_{j}$ at $\tau=0$ and as $\tau \rightarrow \infty$}

Seeing that $K \geq 1, N \in \mathbb{N}>1$ and $\mu \in \mathbb{R}^{+}$, we have that the characteristic function $P_{j}(\lambda, \tau, \eta)$ in 3.9$)$ when $\tau=0$ becomes

$$
P_{j}(\lambda, 0, \eta)=\lambda^{2}+\mu \lambda+K \mu\left(1-\cos \left(2 x_{1}^{*}\right)\right)+\frac{K \mu}{N-1}\left(1+\cos \left(2 x_{1}^{*}\right)\right)=0,
$$

whose roots are

$$
\lambda_{ \pm}=-\frac{\mu}{2} \pm \frac{1}{2}\left(\mu^{2}-4 K \mu\left[1-\cos \left(2 x_{1}^{*}\right)+\frac{1}{N-1}\left(1+\cos \left(2 x_{1}^{*}\right)\right)\right]\right)^{1 / 2}
$$

since $\left|\cos \left(2 x_{1}^{*}\right)\right|<1$, see 3.10 , the discriminant is always smaller than $\mu^{2}$; consequently, $\operatorname{Re}\left(\lambda_{ \pm}\right)<0$. 
When $\tau \rightarrow \infty$, assuming $\operatorname{Re}(\lambda)>0$ in 3.9 , we obtain,

$$
\lim _{\tau \rightarrow \infty} \lambda_{ \pm}=-\frac{\mu}{2} \pm \frac{1}{2}\left(\mu^{2}-4 K \mu\left(1-\cos \left(2 x_{1}^{*}\right)\right)\right)^{1 / 2}
$$

here again the discriminant is always smaller than $\mu^{2}$; thus, $\operatorname{Re}\left(\lambda_{ \pm}\right)<0$, which contradicts the assumption $\operatorname{Re}(\lambda)>0$, and the roots of $P_{j}$ are therefore not on the right-hand side of the complex plane when $\tau \rightarrow \infty$. These results are valid for both equilibria $x_{1}^{*}=\phi^{ \pm}$.

\subsubsection{Conditions for the existence of symmetry-breaking bifurc- ations}

For the characteristic function $P_{j}$ in $(3.9)$, following $(2.12)$, we have

$$
\begin{aligned}
R(\lambda, \eta) & =\lambda^{2}+\mu \lambda+K \mu\left(1-\cos \left(2 x_{1}^{*}\right)\right) \\
S(\lambda, \eta) & =\frac{K \mu}{N-1}\left(1+\cos \left(2 x_{1}^{*}\right)\right),
\end{aligned}
$$

and substituting $\lambda=\mathrm{i} \omega$ we obtain

$$
\begin{aligned}
R(\mathrm{i} \omega, \eta) & =-\omega^{2}+K \mu\left(1-\cos \left(2 x_{1}^{*}\right)\right)+\mathrm{i} \mu \omega \\
S(\mathrm{i} \omega, \eta) & =\frac{K \mu}{N-1}\left(1+\cos \left(2 x_{1}^{*}\right)\right),
\end{aligned}
$$

thus the polynomial $F(\omega)$ from $(2.15)$ becomes

$$
\begin{aligned}
F(\omega)= & \omega^{4}+\left(\mu^{2}-2 K \mu\left(1-\cos \left(2 x_{1}^{*}\right)\right)\right) \omega^{2} \\
& +(K \mu)^{2}\left(1-\cos \left(2 x_{1}^{*}\right)\right)^{2}-\left(\frac{K \mu}{N-1}\right)^{2}\left(1+\cos \left(2 x_{1}^{*}\right)\right)^{2}=0,
\end{aligned}
$$

from which we get

$$
\begin{aligned}
\omega_{ \pm}^{2}= & -\frac{1}{2}\left(\mu^{2}-2 K \mu\left(1-\cos \left(2 x_{1}^{*}\right)\right)\right) \\
& \pm \frac{1}{2}\left[\left(\mu^{2}-2 K \mu\left(1-\cos \left(2 x_{1}^{*}\right)\right)\right)^{2}\right. \\
& \left.-4\left\{(K \mu)^{2}\left(1-\cos \left(2 x_{1}^{*}\right)\right)^{2}-\left(\frac{K \mu}{N-1}\right)^{2}\left(1+\cos \left(2 x_{1}^{*}\right)\right)^{2}\right\}\right]^{1 / 2} .
\end{aligned}
$$

For the sake of simplicity we write

$$
\omega_{ \pm}=\sqrt{-\frac{b}{2} \pm \frac{1}{2} \sqrt{b^{2}-4 c}},
$$

where

$$
\begin{aligned}
& b=\mu^{2}-2 K \mu\left(1-\cos \left(2 x_{1}^{*}\right)\right) \\
& c=(K \mu)^{2}\left(1-\cos \left(2 x_{1}^{*}\right)\right)^{2}-\left(\frac{K \mu}{N-1}\right)^{2}\left(1+\cos \left(2 x_{1}^{*}\right)\right)^{2} .
\end{aligned}
$$


The first necessary condition for the existence of bifurcations in $W_{j}$ is 3.18 , then substituting $b$ and $c$ in this condition we obtain

$$
\mu^{2}-4 \mu K\left(1-\cos \left(2 x_{1}^{*}\right)\right)+\left(\frac{2 K}{N-1}\right)^{2}\left(1+\cos \left(2 x_{1}^{*}\right)\right)^{2} \geq 0 .
$$

By calculating the real roots of this equation, it is possible to find the boundaries in which this inequality holds true; these real roots follow two curves depending on $K$ with $N$ as parameter,

$$
\begin{aligned}
& \mu_{ \pm}(K ; N)= \\
& 2 K\left(1-\cos \left(2 x_{1}^{*}\right)\right) \pm 2 K\left[\left(1-\cos \left(2 x_{1}^{*}\right)\right)^{2}-\left(\frac{1}{N-1}\right)^{2}\left(1+\cos \left(2 x_{1}^{*}\right)\right)^{2}\right]^{1 / 2}
\end{aligned}
$$

Note that the discriminant is always smaller than the square of the first term; therefore, $\mu_{ \pm} \in \mathbb{R}^{+}$. The set $M$ of all values $\mu$ satisfying condition (3.18) is

$$
M=\left\{\mu \in \mathbb{R}^{+} / \mu \in\left\{\left\langle 0, \mu_{-}\right] \cup\left[\mu_{+},+\infty\right\rangle\right\}\right\} .
$$

Additional necessary conditions for the existence of Hopf bifurcations are given in Lemma 3.1. Condition $b \geq 0$ is equivalent to

$$
\mu \geq 2 K\left(1-\cos \left(2 x_{1}^{*}\right)\right)
$$

and condition $c>0$ means

$$
(N-1)^{2}\left(1-\cos \left(2 x_{1}^{*}\right)\right)^{2}-\left(1+\cos \left(2 x_{1}^{*}\right)\right)^{2}>0,
$$

here, since all factors are greater than zero, this is equivalent to

$$
\frac{N-2}{N}>\cos \left(2 x_{1}^{*}\right)
$$

Now, we will analyze conditions for the existence of bifurcations in $W_{j}$ considering three cases:

- When $K=1$, we have $\cos \left(2 x_{1}^{*}\right)=0$, see (3.10); thus, the following analysis will be valid for both equilibria $x_{1}^{*}=\phi_{1}^{ \pm}$. For this case, curves $\mu_{ \pm}$from 3.35 become

$$
\mu_{ \pm}(1 ; N)=2 \pm \frac{2}{N-1} \sqrt{N(N-2)}
$$

clearly, for $N \in \mathbb{N}>1$ we obtain

$$
0<\mu_{-}(1 ; N) \leq 2 \leq \mu_{+}(1 ; N)
$$


From 3.38 we see that $c \geq 0$ is always true for this case. From Lemma 3.1 , 2b, we know from (3.34) that $b$ has to be negative for real solutions $\omega$ of (3.31) to exist, this condition is equivalent to

$$
\mu<2
$$

thus, from 3.36), 3.40 and (3.41), we see that these bifurcations in $W_{j}$ can appear if and only if

$$
\mu \in\left(0, \mu_{-}\right]
$$

- For the equilibrium $x_{1}^{*}=\phi^{+}$, with $K>1$, we have $\cos \left(2 x_{1}^{*}\right)=\frac{1}{K} \sqrt{K^{2}-1}$. We know this equilibrium is unstable in $\operatorname{Fix}\left(\mathbf{S}_{N}\right)$, see sections 3.2 .1 and 3.2 .2 . Curves $\mu_{ \pm}$in 3.35 for this case become

$$
\begin{aligned}
& \mu_{ \pm}(K ; N)= \\
& 2\left(K-\sqrt{K^{2}-1}\right) \pm 2\left[\left(K-\sqrt{K^{2}-1}\right)^{2}-\frac{1}{(N-1)^{2}}\left(K+\sqrt{K^{2}-1}\right)^{2}\right]^{1 / 2} .
\end{aligned}
$$

At this point, it is better to express conditions (3.37) and 3.38) in terms of the curves $b=0$ and $c=0$. Curve $b=0$ is equivalent to

$$
\mu=\mu_{b}(K):=2\left(K-\sqrt{K^{2}-1}\right)
$$

and curve $c=0$ is equivalent to

$$
K(N)=\mathcal{K}_{N}:=\frac{N}{2} \sqrt{\frac{1}{N-1}}
$$

In figure 3.6, curves from (3.43), 3.44) and (3.45) are shown for various values of $N$. Curves $\mu_{+}(K ; N)$ are shown in dotted lines and curves $\mu_{-}(K ; N)$ in dashed lines; curve $\mu_{b}(K)$ is the solid black line. Here conditions $b>0$ and $b<0$ correspond to the regions above and below curve $\mu_{b}$ respectively; conditions $c<0$ and $c>0$ are identified with the right side and left side of each vertical line $\mathcal{K}_{N}$. Let us take as an example $N=2$; from 3.45 we see that $\mathcal{K}_{2}=1$, but we know that $K$ has to be greater than one; thus, bifurcations can occur at the right-hand side of the vertical line $K=\mathcal{K}_{2}$, where $c<0$. From the additional conditions for the existence of bifurcations given in Lemma 3.1, we see that with $c<0$, bifurcations occur only for $\omega_{+}$; the two curves $\mu_{ \pm}$in 3.43 are, for this case, in $\mathbb{C} \backslash \mathbb{R}$; therefore, bifurcations can occur for all $\mu>0$. 


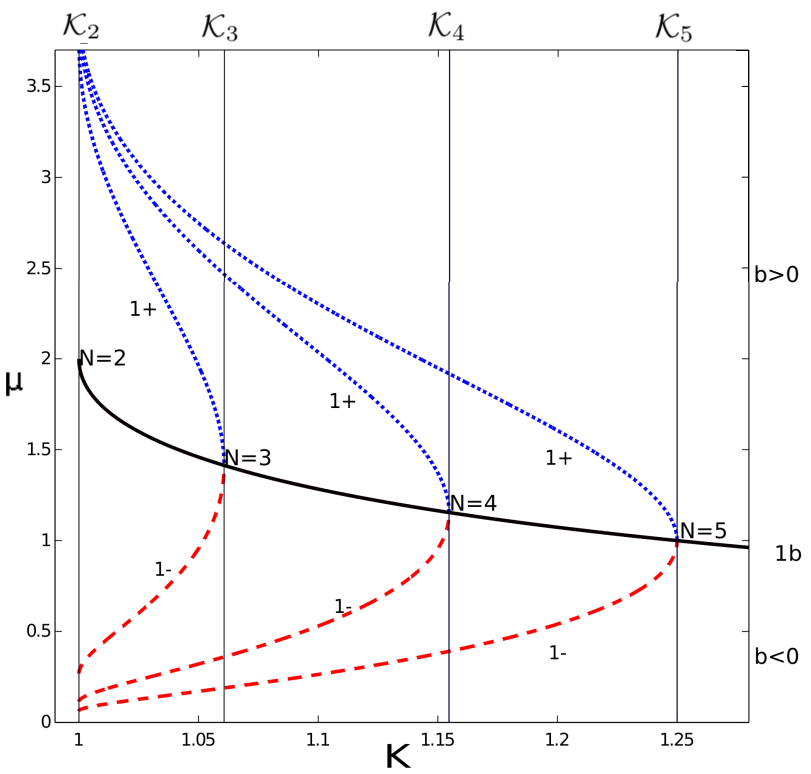

Figure 3.6: Curves showing conditions for the existence of symmetry-breaking bifurcations with $x_{1}^{*}=\phi^{+}$and $K>1$.

- For the equilibrium $x_{1}^{*}=\phi^{-}$with $K>1$ the curves $\mu_{ \pm}$from 3.35 become

$$
\begin{aligned}
& \mu_{ \pm}= \\
& 2\left(K+\sqrt{K^{2}-1}\right) \pm 2\left[\left(K+\sqrt{K^{2}-1}\right)^{2}-\frac{1}{(N-1)^{2}}\left(K-\sqrt{K^{2}-1}\right)^{2}\right]^{1 / 2},
\end{aligned}
$$

and the condition $c>0$ from 3.38 becomes

$$
\frac{N-2}{N}>-\frac{1}{K} \sqrt{K^{2}-1}
$$

which is always true since $K>1$ and $N \in \mathbb{N}>1$. From conditions for existence of bifurcations in $W_{j}$ given in Lemma 3.1, we see that for $c>0$ we need $b<0$ in order for bifurcations to occur, i.e.,

$$
\mu<2\left(K+\sqrt{K^{2}-1}\right)
$$

therefore, from (3.46) and 3.47), we see that bifurcations can occur in $W_{j}$ at the equilibrium $x_{1}^{*}=\phi^{-}$with $K>1$ if

$$
\mu \in\left(0, \mu_{-}\right)
$$

This also means that bifurcations can occur for both $\omega_{ \pm}$.

The analysis of roots of the polynomial $F(\omega)$ from 3.31 in the above paragraphs gives us necessary conditions for the existence of roots of $P_{j}(\mathrm{i} \omega, \tau, \eta)=0$, i.e. $\omega \in \mathbb{R}^{+}$. However, 
it is necessary to also impose conditions on $\sin (\omega \tau)$ and $\cos (\omega \tau)$ given in section 2.5 , to determine at which time delays the linearization at the equilibrium has imaginary eigenvalues. From (2.14) and 3.30 we have

$$
\begin{aligned}
\sin \left(\omega_{ \pm} \tau\right) & =\frac{\omega_{ \pm}(N-1)}{K\left(1+\cos \left(2 x_{1}^{*}\right)\right)} \\
\cos \left(\omega_{ \pm} \tau\right) & =\frac{\left(\omega_{ \pm}^{2}-K \mu\left(1-\cos \left(2 x_{1}^{*}\right)\right)\right)(N-1)}{K \mu\left(1+\cos \left(2 x_{1}^{*}\right)\right)} .
\end{aligned}
$$

Note that the denominator in those terms does not vanish for with $K, \mu \in \mathbb{R}^{+}, K \geq 1$, $N \in \mathbb{N}>1$, since $\cos \left(2 x_{1}^{*}\right)$ from $\left(3.10\right.$ satisfies $\left|\cos \left(2 x_{1}^{*}\right)\right|<1$. At this point, we can calculate the time delays $\tau \geq 0$ associated to $\omega_{ \pm}$using (2.16), which for this case becomes

$$
\tau_{ \pm}(\mu, K ; n)=\frac{1}{\omega_{ \pm}}\left(\operatorname{Arctan}\left(\frac{\omega_{ \pm} \mu}{\omega_{ \pm}^{2}-K \mu\left(1-\cos \left(2 x_{1}^{*}\right)\right)}\right)+2 n \pi\right), \quad n \in \mathbb{N}
$$

$\omega_{ \pm}$is computed using 3.32 . The last necessary condition for the existence of bifurcation points is the transversality condition, given in equation (2.18) and (2.19), where

$$
\begin{aligned}
& A=\omega_{ \pm}^{2} \mu \\
& B=\omega_{ \pm}\left(\omega_{ \pm}^{2}-K \mu\left(1-\cos \left(2 x_{1}^{*}\right)\right)\right) \\
& C=\mu-\tau\left(\omega_{ \pm}^{2}-K \mu\left(1-\cos \left(2 x_{1}^{*}\right)\right)\right) \\
& D=\omega_{ \pm}(2+\tau \mu)
\end{aligned}
$$

As we already know, the sign of $\delta(\mu, K ; N)$ determines if the root crosses the imaginary axis from the left to the right or vice versa, this sign depends on numerator $A C+D B$, thus using (3.32), (3.48), and (3.50), we obtain

$$
A C+D B=2 \omega_{ \pm}^{4}+\omega_{ \pm}^{2}\left(\mu^{2}-2 K \mu\left(1-\cos \left(2 x_{1}^{*}\right)\right)\right)
$$

From $(3.33)$ and $(3.34)$, we see that

$$
A C+D B= \pm \omega_{ \pm}^{2} \sqrt{b^{2}-4 c}
$$

here we use $\omega_{ \pm}$instead of $\omega$ to bring out that there are two possible values for $\omega$; we can express $\delta$ as a function of $\omega_{ \pm}(\mu, K ; N)$, thus the sign of $\delta$ is

$$
\operatorname{sgn}\left(\delta\left(\omega_{ \pm}\right)\right)=\left\{\begin{array}{lll}
+1 & \text { for } & \omega_{+} \\
-1 & \text { for } & \omega_{-}
\end{array} .\right.
$$

The latter equation implies that when bifurcation occurs, whenever $\omega_{-} \in \mathbb{R}^{+}$exists, root $\lambda=\mathrm{i} \omega_{-}$crosses the imaginary axis from the right to the left, whereas whenever $\omega_{+} \in \mathbb{R}^{+}$ exists, root $\lambda=i \omega_{+}$crosses the imaginary axis from the left to the right. 


\subsubsection{Curves of symmetry-breaking bifurcations}

In the previous section we analyzed conditions for the existence of symmetry-breaking bifurcations in terms of parameters $\mu, K \in \mathbb{R}^{+}$with $K \geq 1$ and $N \in \mathbb{N}>1$ for both equilibria $x_{1}^{*}=\phi^{ \pm}$. In this section we will obtain bifurcation curves for $W_{j}$ using the $S_{n}$ map, see section 2.5, and we shall compare these curves with those obtained for $\operatorname{Fix}\left(\mathbf{S}_{N}\right)$ in section 3.2

We will consider three cases:

- When $K=1$, for both equilibria $x_{1}^{*}=\phi^{ \pm}$. We already know that bifurcations can occur only for $\mu<2$, see $(3.41)$; thus the frequency $\omega$ in $(3.32)$ becomes

$$
\omega_{ \pm}^{2}=-\frac{1}{2} \mu(\mu-2) \pm \frac{1}{2} \mu\left[(\mu-2)^{2}-4\left(1-\frac{1}{(N-1)^{2}}\right)\right]^{1 / 2},
$$

and here we distinguish two cases:

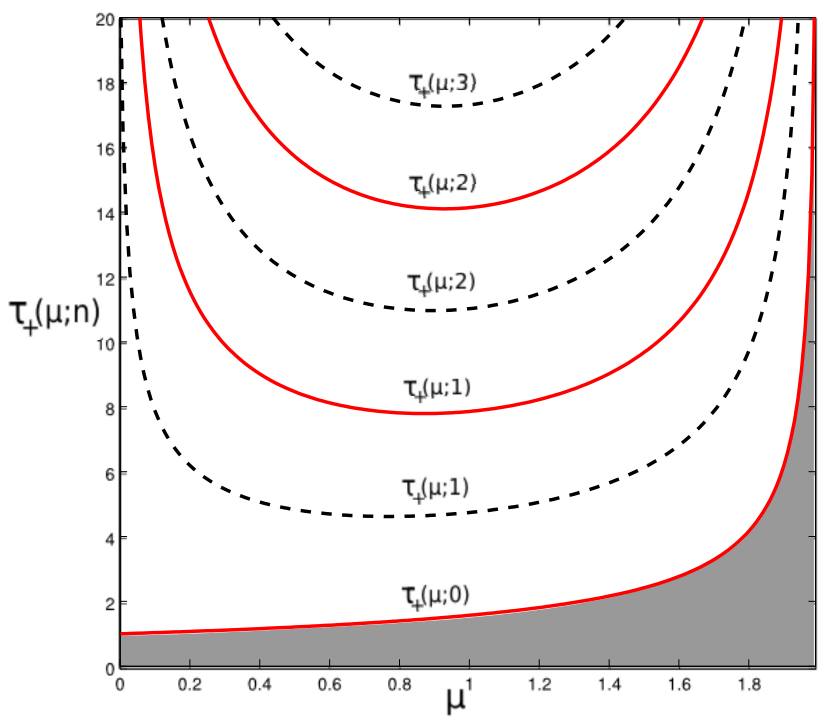

Figure 3.7: Symmetry-preserving bifurcation curves in dashed lines and symmetrybreaking bifurcation curves in solid line for both equilibria $x_{1}^{*}=\phi^{ \pm}$with $N=2$ and $K=1$; bifurcations occur with roots crossing imaginary axis from the left to the right. There are no roots on the right-hand side of the complex plane within the shadowed region. However, there are constant zero roots.

- When $N=2$, we have $\omega_{+}=\sqrt{\mu(2-\mu)}$ and $\omega_{-}=0$, and from (3.51) we know that bifurcations associated to $\omega_{+}$cross the imaginary axis from the left to the 
right. Then, plotting the curves for $\tau_{+}(\mu, 1 ; n)$ using 3.49 , and comparing them with those curves obtained for $\operatorname{Fix}\left(\mathbf{S}_{N}\right)$, see figure 3.1, we obtain the curves shown in figure 3.7 curves for $\tau_{+}$corresponding to $W_{j}$ are plotted in solid lines and curves for $\tau_{+}$corresponding to $\operatorname{Fix}\left(\mathbf{S}_{N}\right)$ are in dashed lines, with the time-delay as a bifurcation parameter; note that each curve indicates a new root crossing the imaginary axis from the left to the right. Therefore, within the shadowed region, there are no roots on the right-hand side of the complex plane; however, due to zero eigenvalues in $\operatorname{Fix}\left(\mathbf{S}_{N}\right)$ and in $W_{j}$ nothing can be said about the stability of the equilibria in this region.
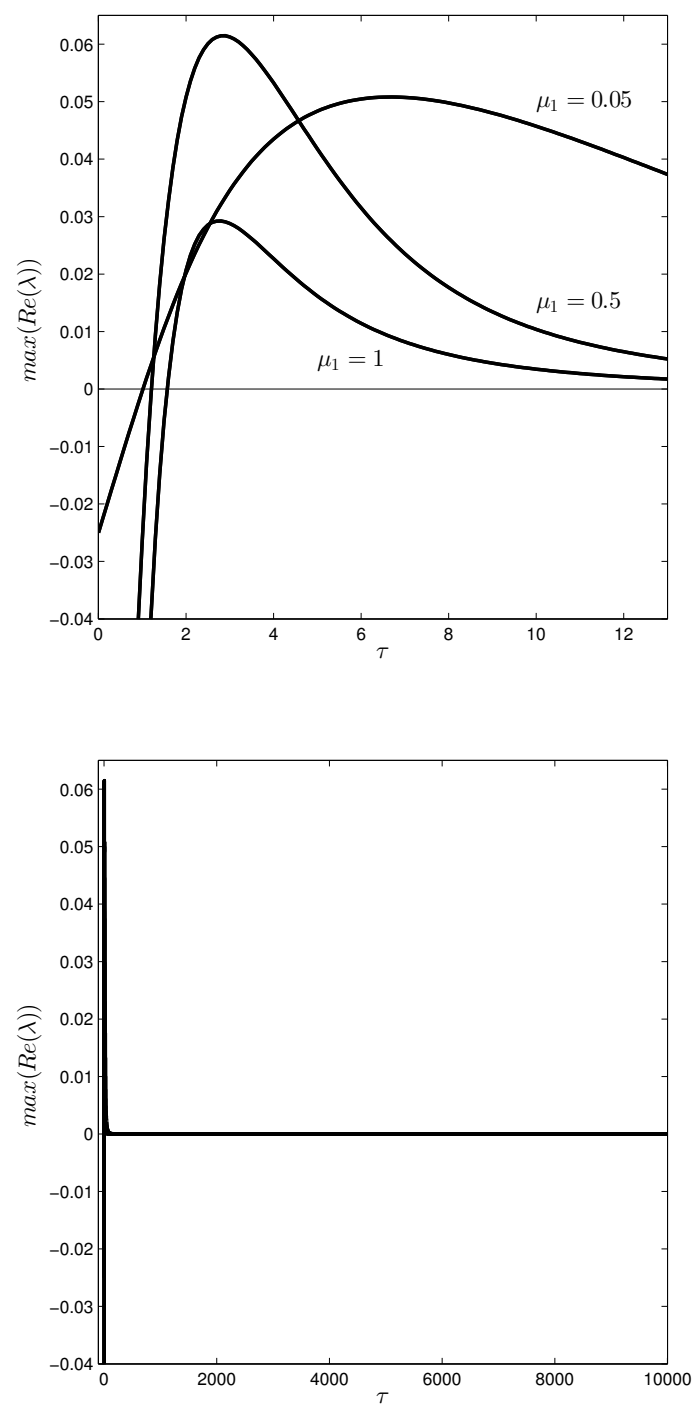

Figure 3.8: Real part of the rightmost root for the function $P_{j}$, for $N=2$, $K=1$ and $\mu=\{0.05,0.5,1\}$.

In figure 3.8, the real part of the rightmost root for the case $N=2, K=1$ 
and $\mu=\{0.05,0.5,1\}$ was computed as a function of the time-delay using the Lambert W function, with both Newton's and Halley's schemes, see section 2.4. it can be seen that this root crosses the imaginary axis at a low value of $\tau$ (approx. $\tau=1$ ), and never comes back, but it approaches zero as $\tau \rightarrow \infty$.

- When $N>2$, we know from 3.39 and 3.42 that bifurcations can occur for $\mu \leq \mu_{-}(1 ; N)<2$ and, from (3.53), we see that both $\omega_{ \pm} \in \mathbb{R}^{+}$. We also know, by looking at the sign of $\delta\left(\omega_{ \pm}\right)$in $(3.52)$, the direction in which these roots cross the imaginary axis. In figure 3.9 curves $\tau_{ \pm}$as a function of $\mu$, from 3.49 , for $W_{j}$ with $N=3$ and for $\operatorname{Fix}\left(\mathbf{S}_{N}\right)$ from 3.27$)$ are shown. For $\operatorname{Fix}\left(\mathbf{S}_{N}\right)$, these curves are shown in solid line; as we already saw in section 3.2.3, these roots cross the imaginary axis from the left to the right. Curves for $W_{j}$ are shown in dotted line for $\tau_{+}$and in dashed line for $\tau_{-}$. The value of $\mu_{-}(1 ; N)$ for $N=3$ bounding the curves $\tau_{ \pm}$, is also shown, see 3.39 . Within the shadowed region, there are no roots with positive real parts; however, there is a zero root of $P_{\mathrm{Fix}\left(\mathbf{S}_{N}\right)}$.

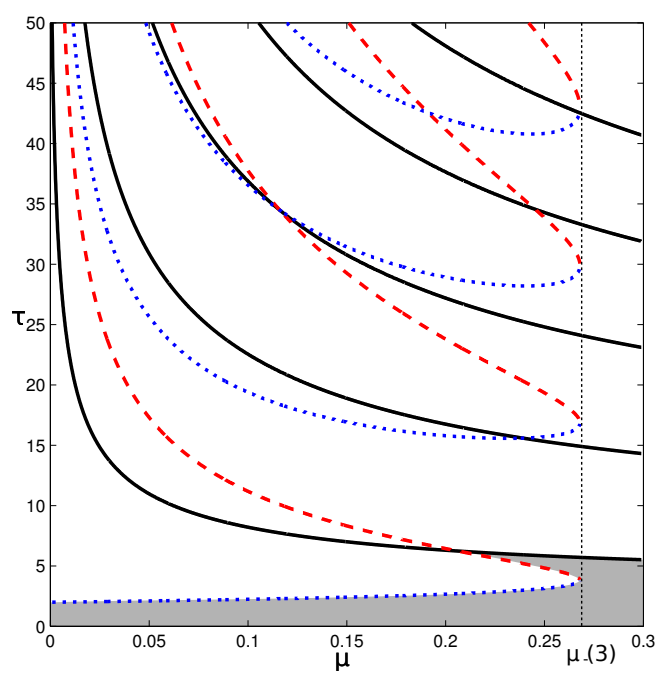

Figure 3.9: Symmetry-preserving bifurcation curves in solid line, see (3.27), and symmetry-breaking bifurcation curves in dotted line for $\mu_{+}$and in dashed line for $\mu_{-}$, see (3.43). These curves are valid for both equilibria $x_{1}^{*}=\phi^{ \pm}$, for $N=3$ and $K=1$. Within the shadowed region, there are no roots with positive real part.

- If $K>1$, the equilibrium $x_{1}^{*}=\phi^{+}$is, as we saw in section 3.2.1, unstable in $\operatorname{Fix}\left(\mathbf{S}_{N}\right)$, 
but we can find bifurcations of this unstable equilibrium in $W_{j}$. From Lemma 3.1 and $(3.34)$ we see that

- If $c \leq 0$ then $\omega_{+} \in \mathbb{R}_{0}^{+}$.

- If $c>0$ and $b<0$ then $\omega_{ \pm} \in \mathbb{R}^{+}$.

Condition $b<0$ implies

$$
\mu<2\left(K-\sqrt{K^{2}-1}\right)<2
$$

and condition $c>0$ is equivalent to

$$
K<\mathcal{K}_{N}
$$

where $\mathcal{K}_{N}$ is defined in (3.45). For a given $N$, we see that for small values of $\mu$ and $K$ bifurcations associated to $\omega_{ \pm}$(roots crossing the imaginary axis in both directions) are possible; however, for $K \geq \mathcal{K}_{N}$ only bifurcations related to $\omega_{+}$appear, i.e., roots crossing the imaginary axis from the left to the right.

- If $K>1$, the equilibrium $x_{1}^{*}=\phi^{-}$is stable in $\operatorname{Fix}\left(\mathbf{S}_{N}\right)$ at $\tau=0$, see section (3.2.1). In figure 3.10, curves $\tau_{ \pm}(\mu)$ for both $\operatorname{Fix}\left(\mathbf{S}_{N}\right)$ and $W_{j}$ are shown.

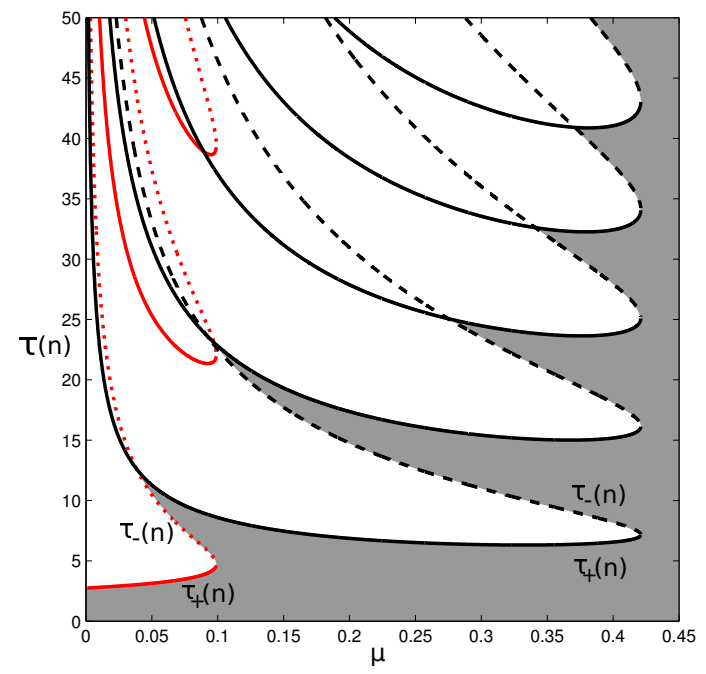

Figure 3.10: For the equilibrium $x_{1}^{*}=\phi^{-}$with $N=3$ and $K=1.05$ curves of symmetry-preserving bifurcations are shown on the right side in dashed/solid black lines and curves of symmetry-breaking bifurcations are shown on the left side in dotted/solid red lines. Within the shadowed regions, the system remains stable. 


\subsection{Equivariant Hopf bifurcations for $N=2,3$}

If we restrict the full-phase model in (3.1) to two nodes $(N=2)$, it is not difficult to see that the resulting model has $\mathbf{S}_{2}=\mathbb{Z}_{2}$ as a symmetry group. In this case, purely imaginary roots of $P_{\mathrm{Fix}\left(\mathbf{S}_{2}\right)}$ lead to symmetry-preserving Hopf bifurcation of fully-synchronized periodic orbits with spatial symmetry group $K=\mathbf{S}_{2}$, see section 2.2.1. Purely imaginary roots of $P_{1}$ lead to symmetry-breaking Hopf bifurcation of periodic orbits with $\mathbb{Z}_{2}$ spatiotemporal symmetry and trivial spatial symmetry, i.e., the first and the second oscillators are half a period out of phase. This follows from the Equivariant Hopf Theorem; for details, see Golubitsky et al. (1988).

For three nodes $(N=3)$, the full-phase model has $\mathbf{S}_{3}=\mathbf{D}_{3}$ as a symmetry group where $\mathbf{D}_{m}$ is the dihedral group of order $2 m$ (rotation and reflections in the plane). In this case, purely imaginary roots of $P_{\mathrm{Fix}\left(\mathbf{S}_{3}\right)}$ lead to symmetry-preserving Hopf bifurcation of fully-synchronized periodic orbits with spatial symmetry group $K=\mathbf{S}_{3}$ as before. Purely imaginary roots of $P_{1}$ lead to symmetry-breaking Hopf bifurcation of two families of periodic orbits (modulo symmetry), one with $H=\mathbb{Z}_{3}$ as spatio-temporal symmetry group, corresponding to coordinate shifts $i \rightarrow i+1 \bmod 3$ and trivial spatial symmetry group, and one with with $K=\mathbb{Z}_{2}\left(\pi_{1,2}\right)$-spatial symmetry and $H / K=\mathbb{Z}_{2}\left(\pi_{1,3}\right)$ as spatiotemporal symmetry group; for details, see Golubitsky et al. (1988). For the first family of periodic orbits, the oscillators are $1 / 3$ of a period out of phase. In the second family of periodic orbits, the first and the second oscillators are in phase and oscillate with twice

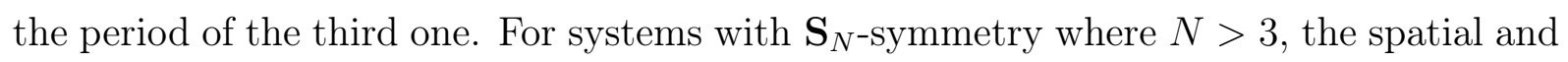
spatio-temporal symmetry groups of bifurcating periodic orbits in the case of equivariant Hopf bifurcation having been classified in Dias and Rodrigues (2009). 


\section{Chapter 4}

\section{Phase model}

In the previous chapter, an analysis of bifurcations emerging from equilibria in a $N$-node fully connected network using the so-called full-phase was made. In this chapter, the same analysis will be applied, but using the instantaneous phase $\theta(t)$ instead of the full-phase $\phi(t)$. As in the classical approach to the PLL analysis the double-frequency term will be neglected. The model for the $i$-th node can be obtained following Bueno, Ferreira, and Piqueira (2009); Piqueira et al. 2006)

$$
\ddot{\theta}_{i}(t)+\mu \dot{\theta}_{i}(t)-\frac{K \mu}{N-1} \sum_{j=1, j \neq i}^{N} \sin \left(\theta_{j}(t-\tau)-\theta_{i}(t)-\omega_{M} \tau\right)=0, \quad i=1, \ldots, N .
$$

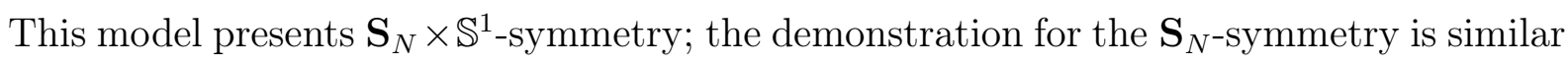
to the full-phase model and will be omitted here. The translational $C \in \mathbb{S}^{1}=\mathbb{R} /(2 \pi \mathbb{Z})$ acts on $\theta \in \mathbb{T}^{N}, \mathbb{T}^{N}=(I \mathbb{R} / 2 \pi \mathbb{Z})^{N}$ as $\theta_{j} \rightarrow\left(\theta_{j}+C\right) \bmod 2 \pi, j=1, \ldots, N$, and it is not difficult to see that if $\left.\theta(t)=\left[\theta_{1}(t), \ldots, \theta_{N} t\right)\right]^{T}$ is a solution to 4.1), then any translated solution $\tilde{\theta}(t)$ with $\tilde{\theta}_{j}(t)=\theta_{j}(t)+C$ is also a solution which proves that 4.1 has translation symmetry. This system has $\mathbf{S}_{N}$-invariant relative equilibria

$$
\theta_{k}(t)=\Omega(\tau) t+\theta^{0}, \quad k=1, \ldots, N
$$

where the rotation frequency $\Omega(\tau)$ satisfies

$$
\Omega(\tau)=-K \sin \left(\left(\Omega(\tau)+\omega_{M}\right) \tau\right)
$$

and $\theta^{0}$ is an arbitrary constant; although $\Omega$ depends on $\omega_{M}, K$ and $\tau$, we write $\Omega(\tau)$ because we are interested in the time-delay as a bifurcation parameter.

The $\mathbb{S}^{1}$-symmetry introduced by the simplification of the double-frequency term generates a zero eigenvalue in the characteristic function for the linearized system around 
any equilibrium or relative equilibrium. The $\mathbf{S}_{N}$-symmetry allows us to find a block decomposition of the linearization at a relative equilibrium as we did in Chapter 3. Remark. Relative equilibria which are not $\mathbf{S}_{N^{-}}$invariant might exist if $\theta_{i}(t)=\Omega(\tau) t+\theta_{i}^{0}$ for all $i$, but $\theta_{i}^{0} \neq \theta_{j}^{0}$, but this case is not studied here.

In a rotating frame with frequency $\Omega(\tau)$ such that

$$
\vartheta_{k}(t)=\theta_{k}(t)-\Omega(\tau) t-\theta^{0}, \quad k=1, \ldots, N
$$

setting $\theta^{0}=0$ for simplicity, we can rewrite equation 4.1 with $x_{1}^{(j)}=\theta_{j}$, and $x_{2}^{(j)}=\dot{\theta}_{j}$, as

$$
\begin{aligned}
& \dot{x}_{1}^{(i)}=x_{2}^{(i)} \\
& \dot{x}_{2}^{(i)}=-\mu x_{2}^{(i)}-\mu \Omega(\tau)+\frac{K \mu}{N-1} \sum_{\substack{j=i \\
j \neq 1}}^{N} \sin \left(x_{1 \tau}^{(j)}-x_{1}^{(i)}-\Omega(\tau) \tau-\omega_{M} \tau\right), \\
& i=1, \ldots, N .
\end{aligned}
$$

Then (4.3) takes the form (3.4). Therefore, we will study bifurcation from $\mathbf{S}_{N}$-invariant relative equilibria. Hopf bifurcation from the equilibrium $x=0$ in the comoving frame leads to relative Hopf bifurcation of relative periodic orbits (RPOs), see definition 2.3 .

In our case, $\Gamma=\mathbb{S}^{1} \times \mathbf{S}_{N}$ is compact and any $\operatorname{RPO} \theta(t)$ satisfies $\theta_{j}(t)=\theta_{j}(t-\tau)+C$ for all $j=1, \ldots, N$. Here $\tau \in \mathbb{R}^{+}$is called the relative period of the RPO and $C \in \mathbb{R}$.

Linearising equation (4.3) around its equilibrium point $x=0$ we can compute the matrix $L$ as in 2.6

$$
L x=\left(\begin{array}{c}
x_{2}^{(1)} \\
-K \mu \cos (\widehat{\Omega} \tau) x_{1}^{(1)}-\mu x_{2}^{(1)}+\frac{K \mu}{N-1} \cos (\widehat{\Omega} \tau) e^{-\lambda \tau} \sum_{\substack{i=1 \\
i \neq 1}}^{N} x_{1}^{(i)} \\
\vdots \\
x_{2}^{(N)} \\
-K \mu \cos (\widehat{\Omega} \tau) x_{1}^{(N)}-\mu x_{2}^{(N)}+\frac{K \mu}{N-1} \cos (\widehat{\Omega} \tau) e^{-\lambda \tau} \sum_{\substack{i=1 \\
i \neq N}}^{N} x_{1}^{(i)}
\end{array}\right)
$$

where

$$
\widehat{\Omega}=\Omega(\tau)+\omega_{M},
$$

or

$$
\widehat{\Omega}=-K \sin (\widehat{\Omega} \tau)+\omega_{M}
$$


From 4.2 , we see that

$$
-K \leq \Omega(\tau) \leq K
$$

Now, using the results from section 3.1, particularly (3.5), (3.8), and (3.9) with

$$
\begin{aligned}
q & =K \mu \cos (\widehat{\Omega} \tau) \\
r & =-\frac{K \mu}{N-1} \cos (\widehat{\Omega} \tau) e^{-\lambda \tau}
\end{aligned}
$$

we obtain the characteristic functions

$$
\begin{aligned}
P_{\mathrm{Fix}\left(\mathbf{S}_{N}\right)}(\lambda, \tau, \eta) & \left.=\operatorname{det}\left(\left.\triangle(\lambda, \tau)\right|_{\mathrm{Fix}\left(\mathbf{S}_{N}\right)}\right)\right)=0 \\
& =\lambda^{2}+\mu \lambda+K \mu \cos (\widehat{\Omega} \tau)-K \mu \cos (\widehat{\Omega} \tau) e^{-\lambda \tau}=0 \\
P_{j}(\lambda, \tau, \eta) & =\operatorname{det}\left(\left.\triangle(\lambda, \tau)\right|_{W_{j}}\right)=0 \\
& =\lambda^{2}+\mu \lambda+K \mu \cos (\widehat{\Omega} \tau)+\frac{K \mu}{N-1} \cos (\widehat{\Omega} \tau) e^{-\lambda \tau}=0 .
\end{aligned}
$$

Clearly, $P_{\mathrm{Fix}\left(\mathbf{S}_{N}\right)}$ has a constant zero eigenvalue for all values of $\eta=\left[K, \mu, \widehat{\Omega}, \omega_{M}, N\right]$ due to the translational symmetry. As before, roots in $P_{j}$ have multiplicity $N-1$.

Remark. (Parameter $k_{v}$ in the phase model and the rotating frame) In Correa and Piqueira (2013) a modification of model (4.1) was presented by introducing parameter $k_{v}$ in order to avoid a zero eigenvalue in the characteristic equation $P_{\operatorname{Fix}\left(\mathbf{S}_{N}\right)}(\lambda, \tau, \eta)=0$ in 4.7$)$; the phase model using this new parameter becomes

$$
\ddot{\theta}_{i}(t)+\left(\mu+k_{v}\right) \dot{\theta}_{i}(t)+\mu k_{v} \theta_{i}(t)-\frac{K \mu}{N-1} \sum_{\substack{j=1 \\ j \neq i}}^{N} \sin \left(\theta_{j}(t-\tau)-\theta_{i}(t)-\omega_{M} \tau\right)=0,
$$

$i=1, \ldots, N$. The $\mathbb{R}$-symmetry disappears when $k_{v} \neq 0$. Clearly, there is no point in going into a comoving frame or talking of relative equilibria if there is no translational symmetry; for details and results using this model, see Correa and Piqueira (2013).

\subsection{Symmetry-preserving bifurcations}

In the rotating frame $\vartheta(t)=\theta(t)-\Omega(\tau) t$, the frequency $\Omega(\tau)$ is determined by the nonlinear equation (4.2); thus, for a given $\tau$ there exists a whole family of solutions $\Omega(\tau)$ satisfying this equation; clearly, as $\tau$ increases more solutions appear. We will start analyzing if there are roots at the right side of the complex plane at $\tau=0$; then, from 4.7) when $\tau=0$, we have

$$
P_{\operatorname{Fix}\left(\mathbf{S}_{N}\right)}(\lambda, 0, \eta)=\lambda^{2}+\mu \lambda=0
$$


whose roots are

$$
\lambda_{+}=0, \quad \lambda_{-}=-\mu
$$

Therefore, at $\tau=0, \operatorname{Fix}\left(\mathbf{S}_{N}\right)$ is spectrally stable. In order to find critical delays leading to bifurcations in $\operatorname{Fix}\left(\mathbf{S}_{N}\right)$, we will follow section 2.5, thus using 2.12 , we have that $P_{\text {Fix }\left(\mathbf{S}_{N}\right)}(\lambda, \tau, \eta)=R(\lambda, \tau)+S(\tau) e^{-\lambda \tau}=0$ where

$$
\begin{aligned}
R(\lambda, \tau, \eta) & =\lambda^{2}+\mu \lambda+K \mu \cos (\widehat{\Omega} \tau) \\
S(\tau, \eta) & =-K \mu \cos (\widehat{\Omega} \tau)
\end{aligned}
$$

and from (2.14) we obtain

$$
\begin{aligned}
\sin (\omega \tau) & =-\frac{\omega}{K \cos (\widehat{\Omega} \tau)} \\
\cos (\omega \tau) & =\frac{-\omega^{2}+K \mu \cos (\widehat{\Omega} \tau)}{K \mu \cos (\widehat{\Omega} \tau)},
\end{aligned}
$$

for $K \mu \cos (\widehat{\Omega} \tau) \neq 0$. The polynomial $F(\omega, \tau)$ from 2.15 becomes

$$
F(\omega, \tau)=\omega^{2}\left(\omega^{2}-2 K \mu \cos (\widehat{\Omega} \tau)+\mu^{2}\right)=0
$$

and thus

$$
\omega^{2}=2 K \mu \cos (\widehat{\Omega} \tau)-\mu^{2}, \quad \text { or } \quad \omega=0
$$

From (4.5) we obtain

$$
\cos (\widehat{\Omega} \tau)= \pm \frac{1}{K} \sqrt{K^{2}-\left(\omega_{M}-\widehat{\Omega}\right)^{2}}
$$

thus

$$
\omega= \pm\left(2 \mu \sqrt{K^{2}-\left(\omega_{M}-\widehat{\Omega}\right)^{2}}-\mu^{2}\right)^{1 / 2}, \quad \text { or } \quad \omega=0,
$$

clearly, solutions $\omega \in \mathbb{R}^{+}$exist provided

$$
2 \sqrt{K^{2}-\left(\omega_{M}-\widehat{\Omega}\right)^{2}} \geq \mu
$$

Given $\tau \in \mathbb{R}^{+}$we can compute $\widehat{\Omega}$ using (4.2) and 4.4). For $\omega \in \mathbb{R}^{+}$satisfying (4.11) we compute the $S_{n}$ map, see section 2.5, whose zeroes are the critical bifurcation time delays for $\operatorname{Fix}\left(\mathbf{S}_{N}\right)$. Using 2.18 and 2.19 , we obtain $\delta\left(\omega\left(\tau^{*}\right)\right)$ to find the direction in which roots, if any, cross the imaginary axis. From (4.5), we compute

$$
\widehat{\Omega}_{\tau}^{\prime}=\frac{d \widehat{\Omega}}{d \tau}=-\frac{\widehat{\Omega} K \cos (\widehat{\Omega} \tau)}{1+\tau K \cos (\widehat{\Omega} \tau)} .
$$


and, from 4.8, and 4.9), we have

$$
\begin{aligned}
A & =\frac{\omega^{2} \widehat{\Omega} \sin (\widehat{\Omega} \tau)}{\cos (\widehat{\Omega} \tau)(1+\tau K \cos (\widehat{\Omega} \tau))}+\omega^{2} \mu \\
B & =\omega\left(\omega^{2}-K \mu \cos (\widehat{\Omega} \tau)-\frac{\mu \widehat{\Omega} \sin (\widehat{\Omega} \tau)}{\cos (\widehat{\Omega} \tau)(1+\tau K \cos (\widehat{\Omega} \tau))}\right) \\
C & =\mu+\tau\left(K \mu \cos (\widehat{\Omega} \tau)-\omega^{2}\right) \\
D & =\omega(2+\tau \mu) .
\end{aligned}
$$

\subsubsection{Symmetry-preserving bifurcations from equilibria}

From $(4.2)$, we see that $\Omega(\tau)=0$ is a rotating frame solution and hence relative equilibrium becomes an equilibrium when $\omega_{M} \tau=n \pi$, with $n \in \mathbb{N}_{0}$. Now, here we have two possible cases:

- When $\omega_{M} \tau=2 n \pi$. We have $\widehat{\Omega}=\omega_{M}$ and $\cos (\widehat{\Omega} \tau)=1$, thus from 4.9 we obtain

$$
\begin{aligned}
\sin (\omega \tau) & =-\frac{\omega}{K} \\
\cos (\omega \tau) & =\frac{K \mu-\omega^{2}}{K \mu},
\end{aligned}
$$

and from condition 4.10 we obtain

$$
\omega= \pm \sqrt{2 K \mu-\mu^{2}}
$$

provided $2 K \geq \mu$. Then, from (4.14) and 4.15) we obtain a second condition

$$
\pm \sqrt{2 K \mu-\mu^{2}}=\frac{\omega_{M}}{2 n \pi}\left(\operatorname{Arctan}\left(\frac{\mp \sqrt{2 K \mu-\mu^{2}}}{\mu-K}\right)+2 m \pi\right),
$$

with $n \in \mathbb{N}, m \in \mathbb{Z}$. Here we have two curves $K_{ \pm}\left(\mu ; \omega_{M}, n, m\right)$ for which a critical delay at $\omega_{M} \tau=2 n \pi$ exists with imaginary eigenvalue $\lambda= \pm \mathrm{i} \omega(K, \mu)$ with $\omega$ from 4.15 , and $\widehat{\Omega}=\omega_{M}$. In figure 4.1 curves $K_{+}\left(\mu ; \omega_{M}, n, m\right)$ are shown for $m=\{1, \ldots, 4\}, \omega_{M}=1$ and $n=1$, curves corresponding to $K_{-}$are omitted since do not correspond to $K>0$. Curves $\delta(\mu, K(\mu) ; m)$ which determine the direction in which roots cross the imaginary axis, see (2.18), (2.19) and 4.15), are also shown. From these family of curves periodic orbits bifurcate.

- When $\omega_{M} \tau=(2 n+1) \pi$. We have from condition $4.10 \omega= \pm \mathrm{i} \sqrt{2 K \mu+\mu^{2}}$, but these solutions are disregarded, since we are looking for $\omega \in \mathbb{R}$. 

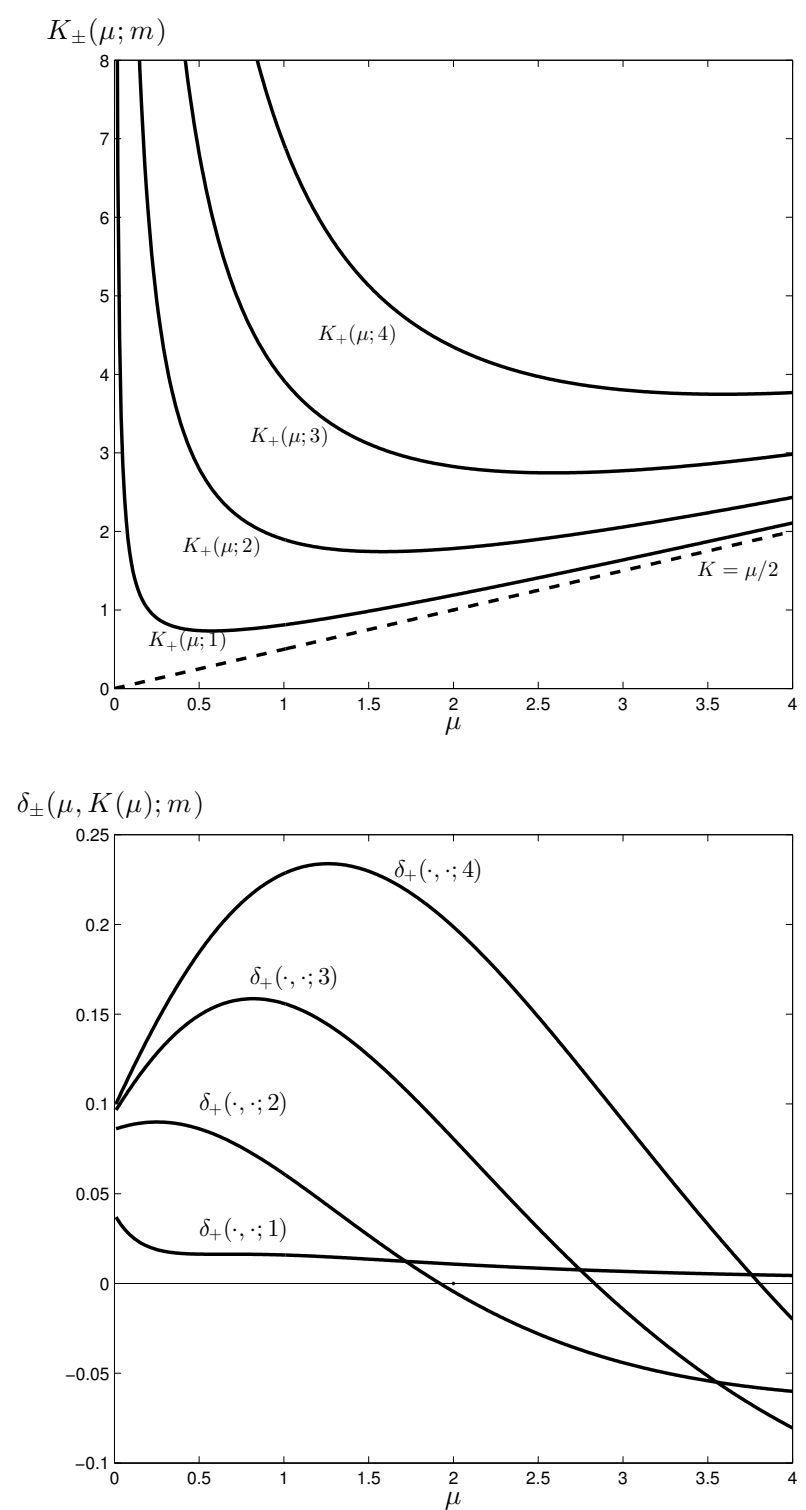

Figure 4.1: Curves $K_{+}\left(\mu ; \omega_{M}, n, m\right)$ from (4.16) and $\delta_{+}(\mu, K(\mu) ; m)$ with $\omega_{M}=1, n=1$ for different values of $m$.

\subsubsection{Symmetry-preserving bifurcations from relative equilibria}

For this case, when $\Omega(\tau) \neq 0$, we carried out numerical computations to find time delays $\tau$ leading to bifurcations in $\operatorname{Fix}\left(\mathbf{S}_{N}\right)$; the procedure is as follows:

- For a given $\tau \in \mathbb{R}^{+}$and parameters $K, \omega_{M}, \mu \in \mathbb{R}^{+}$we calculate all the $m$ real solutions $\widehat{\Omega}^{(j)}=\widehat{\Omega}^{(j)}\left(\tau ; K, \omega_{M}, \mu\right), j=1, \ldots, m$ of 4.5 , noting 4.6) and that the number of solutions is finite and increases with $\tau$.

- For each solution $\widehat{\Omega}^{(j)}$, we compute $\omega_{ \pm}$from 4.11, provided condition 4.12 holds. 
- For each $\omega=\omega_{ \pm}\left(\widehat{\Omega}^{(j)} ; K, \omega_{M}, \mu\right), \omega \in \mathbb{R}^{+}$, we compute the $S_{n}$ map using 4.9 , see section 2.5. since these roots $\omega_{ \pm}$are conjugate pairs, only one of them is necessary, let us say $\omega_{+}$; the $S_{n}$ map gives us the time-delay $\tau_{n}(\tau)$ from $(2.16)$, which depends on the values calculated previously. If $\tau_{n}(\tau)$ matches with the given $\tau$, then we find a bifurcation time-delay, cf. (2.17).

- Finally, in order to determine the direction in which these roots cross the imaginary axis, we compute the sign of $\delta\left(\omega\left(\tau^{*}\right)\right)$ using 2.19 .

In figure 4.2 , the eleven possible curves $\widehat{\Omega}^{(j)}$ within the interval $\tau \in[0,5 \pi]$ are shown with parameters $\mu=1, K=1$ and $\omega_{M}=1$.

In figure 4.3 , the $S_{n}$ maps and all points within the interval $\tau \in[0,5 \pi]$ at which relative periodic orbits bifurcate are shown. The sign for $\delta\left(\omega\left(\tau^{*}\right)\right)$ is positive in all cases.

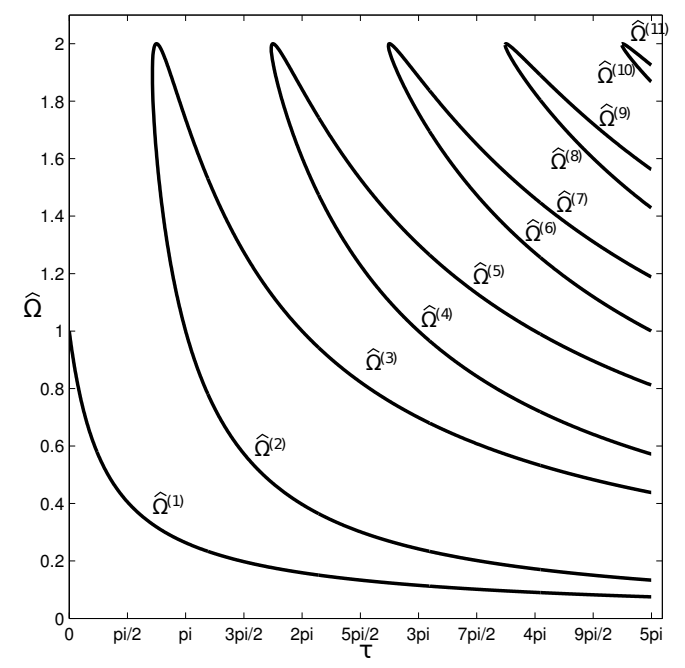

Figure 4.2: Curves $\widehat{\Omega}$ with $\mu=1, K=1$, and $\omega_{M}=1$. 


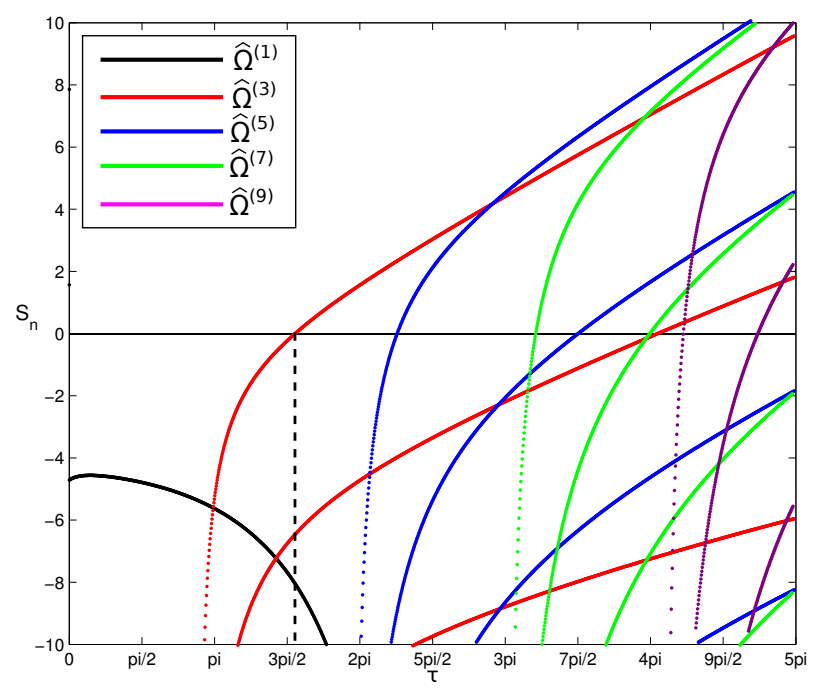

Figure 4.3: $S_{n}$ maps for curves $\widehat{\Omega}^{(j)}$ related to the characteristic function $P_{\operatorname{Fix}\left(\mathbf{S}_{N}\right)}$ in $(4.7)$; in all cases $\delta\left(\omega\left(\tau^{*}\right)\right)$ is positive, i.e., the roots cross imaginary axis from the left to the right. At $S_{n}=0$ along the curves $\widehat{\Omega}^{(j)}$, relative Hopf bifurcation occurs.

\subsection{Symmetry-breaking bifurcations}

As before, we will analyze if there is some unstable root at $\tau=0$ in $P_{j}$; thus, from (4.7) we obtain:

$$
P_{j}(\lambda, 0, \eta)=\lambda^{2}+\mu \lambda+K \mu\left(\frac{N}{N-1}\right)=0
$$

whose roots are

$$
\lambda_{ \pm}=-\frac{\mu}{2} \pm \frac{1}{2}\left(\mu^{2}-4 K \mu \frac{N}{N-1}\right)^{1 / 2}
$$

and since $\mu, K \in \mathbb{R}^{+}$and $N \geq 2$, nonzero roots are always stable. In order to calculate the $S_{n}$ map (see section 2.5, in particular (2.12), we note that by 4.7),

$$
\begin{aligned}
R(\lambda, \tau) & =\lambda^{2}+\mu \lambda+K \mu \cos (\widehat{\Omega} \tau) \\
S(\tau) & =\frac{K \mu}{N-1} \cos (\widehat{\Omega} \tau)
\end{aligned}
$$

hence by (2.14) we obtain

$$
\begin{aligned}
\sin (\omega \tau) & =\frac{\omega(N-1)}{K \cos (\widehat{\Omega} \tau)} \\
\cos (\omega \tau) & =\frac{\left(\omega^{2}-K \mu \cos (\widehat{\Omega} \tau)\right)(N-1)}{K \mu \cos (\widehat{\Omega} \tau)}
\end{aligned}
$$


and polynomial $F$ from 2.15 is

$$
F(\omega, \tau)=\omega^{4}+\left(\mu^{2}-2 K \mu \cos (\widehat{\Omega} \tau)\right) \omega^{2}+\frac{N(N-2)}{(N-1)^{2}} K^{2} \mu^{2} \cos ^{2}(\widehat{\Omega} \tau)=0,
$$

here again, $\widehat{\Omega}=\Omega(\tau)+\omega_{M}$ and $\Omega(\tau)$ satisfies 4.2 . Hence,

$$
\begin{aligned}
\omega_{ \pm}^{2}= & -\frac{1}{2}\left(\mu^{2}-2 K \mu \cos (\widehat{\Omega} \tau)\right) \\
& \pm \frac{1}{2}\left[\left(\mu^{2}-2 K \mu \cos (\widehat{\Omega} \tau)\right)^{2}-4 \frac{N(N-2)}{(N-1)^{2}} K^{2} \mu^{2} \cos ^{2}(\widehat{\Omega} \tau)\right]^{1 / 2} .
\end{aligned}
$$

In 4.19, the discriminant is always smaller than the square of the first term; therefore, so that $\omega \in \mathbb{R}$, this first term has to be non-negative, i.e.,

$$
\mu \leq 2 K \cos (\widehat{\Omega} \tau)
$$

Hence, $\cos (\widehat{\Omega} \tau) \geq 0$. For $\omega \in \mathbb{R}$, the discriminant also has to be greater or equal to zero, i.e.,

$$
2 K \cos (\widehat{\Omega} \tau)-\mu \geq \frac{\sqrt{N(N-2)}}{(N-1)} 2 K \cos (\widehat{\Omega} \tau)
$$

or

$$
\mu \leq 2 K \cos (\widehat{\Omega} \tau)\left(1-\frac{\sqrt{N(N-2)}}{N-1}\right) .
$$

Using (2.19), we obtain $\delta\left(\omega\left(\tau^{*}\right)\right)$ to find the direction in which roots, if any, cross the imaginary axis, from 2.20 and noting 4.13, 4.17), and 4.18 we obtain

$$
\begin{aligned}
A & =\omega_{ \pm}^{2}\left(\frac{\sin (\widehat{\Omega} \tau) \widehat{\Omega}}{\cos (\widehat{\Omega} \tau)(1+\tau K \cos (\widehat{\Omega} \tau))}+\mu\right) \\
B & =\omega_{ \pm}\left(\omega_{ \pm}^{2}-K \mu \cos (\widehat{\Omega} \tau)-\frac{\mu \widehat{\Omega} \sin (\widehat{\Omega} \tau)}{\cos (\widehat{\Omega} \tau)(1+\tau K \cos (\widehat{\Omega} \tau))}\right) \\
C & =\mu-\tau\left(\omega_{ \pm}^{2}-K \mu \cos (\widehat{\Omega} \tau)\right) \\
D & =\omega_{ \pm}(2+\tau \mu)
\end{aligned}
$$

\subsubsection{Symmetry-breaking bifurcations of relative equilibria}

Setting $\lambda=0$ in 4.7 gives

$$
\widehat{\Omega} \tau=\frac{\pi}{2}+n \pi, \quad n \in \mathbb{Z},
$$

where time delays $\tau=\tau^{*}$ correspond to a zero root in $P_{j}$. From 4.5 we see that

$$
\widehat{\Omega}=(-1)^{n+1} K+\omega_{M},
$$


thus, substituting into 4.22 , we obtain

$$
\tau^{*}=\left(\frac{\pi}{2}+n \pi\right) \frac{1}{\omega_{M}+(-1)^{n+1} K}, \quad n \in \mathbb{Z}
$$

such that $\tau^{*} \geq 0$. Now we can calculate $\left.\delta(\omega, \tau)\right|_{\omega=0}$ using 2.18, 4.7) and 4.13,

$$
\delta\left(0, \tau^{*}\right)=\frac{(-1)^{n} K N}{N-1}\left(\omega_{M}+(-1)^{n+1} K\right), \quad n \in \mathbb{Z},
$$

where $n \in \mathbb{Z}$, as in (4.23). At these critical time delays, relative equilibria bifurcate which are not $\mathbf{S}_{N}$-invariant, i.e., they satisfy $\theta_{k}(t)=\Omega(\tau) t+\theta_{k}^{0}$ with $\theta_{k}^{0} \neq \theta_{j}^{0}$ for some $j \neq k$; for details, see Golubitsky et al. (1988), cf. also Section 3.4.

\subsubsection{Symmetry-breaking bifurcations from equilibria}

Now, we shall analyze Hopf bifurcations from equilibria where $\Omega(\tau)=0$ as we did in Section 4.1 .1 for $\operatorname{Fix}\left(\mathbf{S}_{N}\right)$. We know that, for this case, $\omega_{M} \tau=n \pi$ with $n \in \mathbb{N}_{0}$ and $\widehat{\Omega}=\omega_{M} ;$ we thus have two cases:

- When $\omega_{M} \tau=2 n \pi$, Hopf bifurcations with frequencies $\omega_{ \pm}$given by (4.19) are possible if 4.20 holds; note that $\cos (\widehat{\Omega} \tau)=1$. In order to calculate $\delta(\omega, \tau)$ for the case $\tau=2 n \pi / \omega_{M}$ using 2.18 and 4.21 , we compute the denominator $A C+B D$ which determines the sign of $\delta\left(\omega_{ \pm}\right)$as

$$
A C+B D=\omega_{ \pm}^{2}\left(2 \omega_{ \pm}^{2}+\mu^{2}-2 K \mu\right)
$$

but from 4.19 we see that

$$
A C+B D= \pm \omega_{ \pm}^{2} \sqrt{d}
$$

where $d$ denotes the discriminant of 4.19). Hence,

$$
\operatorname{sgn}\left(\delta\left(\omega_{ \pm}\right)\right)=\left\{\begin{array}{lll}
+1 & \text { for } & \omega_{+} \\
-1 & \text { for } & \omega_{-} .
\end{array}\right.
$$

In this case, equivariant Hopf bifurcation takes place and families of non-synchronous periodic orbits bifurcate, see Section 3.4.

- When $\omega_{M} \tau=(2 n+1) \pi$, we have $\cos \left(\omega_{M} \tau\right)=-1$ which violates 4.20 ; therefore, bifurcations are not possible in $W_{j}$ for this case. 


\subsubsection{Curves of symmetry-breaking bifurcations}

For this analysis, we shall follow the steps described in section 4.1 .2 for bifurcations in $\operatorname{Fix}\left(\mathbf{S}_{N}\right)$ and we will continue with the same example. We look for bifurcation points of the characteristic function $P_{j}$ with $\mu=1, K=1, \omega_{M}=1$ choosing $N=2$. Using the $S_{n}$ map as before, critical time delays are shown in figure 4.4. In all cases, $\delta\left(\omega\left(\tau^{*}\right)\right) \geq 0$. The first bifurcation appears at $\tau=\pi$, which is lower than the lowest bifurcation time-delay value in $\operatorname{Fix}\left(\mathbf{S}_{N}\right)$ in figure 4.3 .

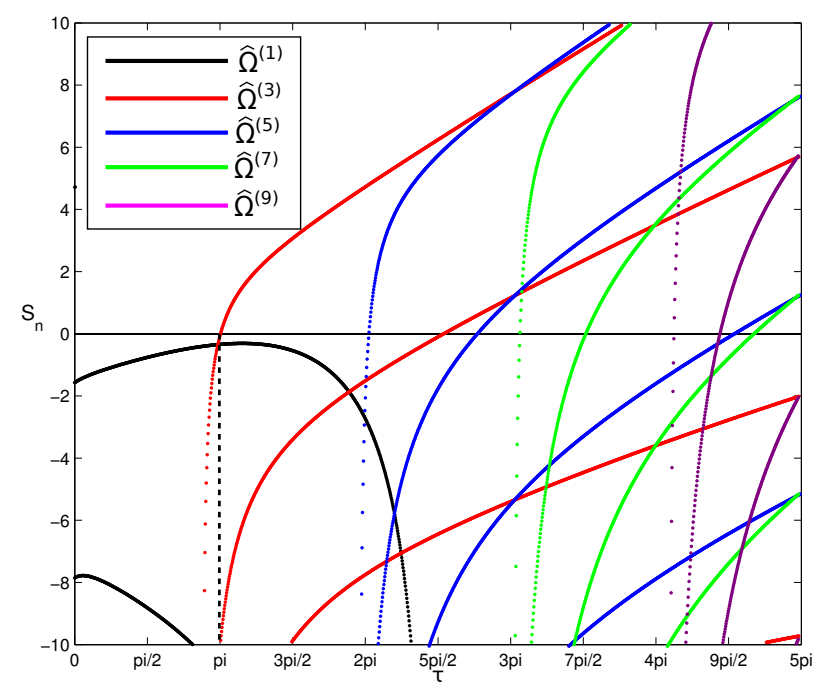

Figure 4.4: $S_{n}$ maps for different curves $\widehat{\Omega}$ related to the characteristic function $P_{j}$ in 4.7); in all cases $\delta\left(\omega\left(\tau^{*}\right)\right) \geq 0$, i.e., roots cross from the left to the right. 


\section{Chapter 5}

\section{Phase-difference model}

In previous chapters we have modelled the network under study, using the two main approaches present in the literature, namely, using the full-phase in chapter 3, and the instantaneous phase in chapter 4. However, in Bueno et al. 2009, 2010); Correa and Piqueira (2013), an alternative approach is used to model a fully connected PLL network using the phase difference between any two nodes $i$ and $j$ which is defined as

$$
\varphi^{(i, j)}(t):=\theta_{i}(t)-\theta_{j}(t-\tau)
$$

From 4.1), using the phase-difference definition, we get

$$
\ddot{\varphi}^{(i, j)}+\mu \dot{\varphi}^{(i, j)}+\frac{K \mu}{N-1}\left[\sum_{l=1, l \neq i}^{N} \sin \left(\varphi^{(i, l)}+\omega_{M} \tau\right)-\sum_{l=1, l \neq j}^{N} \sin \left(\varphi_{\tau}^{(j, l)}+\omega_{M} \tau\right)\right]=0
$$

This phase-difference model has $\mathbf{S}_{N}$-symmetry as it is derived from the phase model of section 4 , but there is no translational symmetry due to the definition of phase-difference.

If $N>2$, then the phase-difference model lives in $\varphi \in \mathbb{R}^{N(N-1)}$ and $N(N-1)>N$ so that the model is expected to have fictitious solutions which do not correspond to the phase model for $\theta \in \mathbb{R}^{N}$ from Section 4 . If $N=2$ and $\theta_{1}([-\tau, 0))$ and $\theta_{2}([-\tau, 0))$ are known, we can compute $\varphi^{(1,2)}$ and $\varphi^{(2,1)}$ for $t=[-\tau, \infty)$, and then compute $\theta(t)$ for $\forall t$ using 5.2 . But from solutions $\left(\varphi^{(1,2)}, \varphi^{(2,1)}\right)$, we cannot reconstruct $\left(\theta_{1}, \theta_{2}\right)$ initial data, and any solution of the $\left(\theta_{1}, \theta_{2}\right)$-dynamics is a solution of $\left(\varphi^{(1,2)}, \varphi^{(2,1)}\right)$-dynamics, but not vice versa. Hence, not all solutions of the $\left(\varphi^{(1,2)}, \varphi^{(2,1)}\right)$-dynamics give solutions of the original $\left(\theta_{1}, \theta_{2}\right)$-dynamics. We demonstrate this issue by studying equilibria of the phase-difference model. 
In $\operatorname{Fix}\left(\mathbf{S}_{N}\right)$, an $N$-node network modelled by (5.1) has a family of $\mathbf{S}_{N}$-invariant equilibria given by

$$
\varphi^{(i, j)}(t)=C, \quad C \in \mathbb{R}
$$

which implies $\theta_{i}(t)-\theta_{j}(t-\tau)=\theta_{j}(t)-\theta_{i}(t-\tau)=C$ for all $i \neq j$. The solutions are relative periodic orbits (RPOs) of the $\theta$-dynamics, i.e., satisfy

$$
\begin{aligned}
& \theta_{i}(t)=\theta_{i}(t-2 \tau)+2 C \\
& \theta_{i}(t)=\theta_{j}(t-\tau)+C .
\end{aligned}
$$

If $C \equiv 0$, then $\theta(t)$ is $2 \tau$-periodic with $\mathbb{Z}_{2}$-spatio temporal symmetry; otherwise the RPOs have $\mathbb{Z}_{2}$-spatio temporal symmetry in a suitable comoving frame.

We can use 5.3 to find solutions to the phase model in chapter 4 restricted to $\operatorname{Fix}\left(\mathbf{S}_{N}\right)$; thus, from 4.1), the $\theta$-dynamics restricted to $\operatorname{Fix}\left(\mathbf{S}_{N}\right)$ is:

$$
\ddot{\theta}_{i}(t)+\mu \dot{\theta}_{i}-K \mu \sin \left(\theta_{i}(t-\tau)-\theta_{i}(t)-\omega_{M} \tau\right)=0
$$

then, by substituting (5.2) into (5.4), we obtain the second-order ODE

$$
\ddot{\theta}_{i}(t)+\mu \dot{\theta}_{i}(t)+K \mu \sin \left(C+\omega_{M} \tau\right)=0
$$

whose solution is

$$
\theta_{j}(t)=-\frac{K}{\mu} \sin \left(C+\omega_{M} \tau\right)(\mu t-1)-\frac{C_{1}}{\mu^{2}}+C_{2} e^{-\mu t}
$$

with $C, C_{1}, C_{2}$ arbitrary constants. From 5.3 we have for any $i \neq j$

$$
\theta_{i}(t)-\theta_{j}(t-\tau)=K \sin \left(\left(C+\omega_{M} \tau\right) \tau\right)+C_{2} e^{-\mu t}\left(1-e^{\mu \tau}\right)
$$

Hence, $C_{2}=0$ and so

$$
C=-K \sin \left(\left(C+\omega_{M} \tau\right) \tau\right)
$$

and so $C=\Omega(\tau) \tau$ with $\Omega(\tau)$ from 4.2 .

Therefore, although the $N$-node model from (5.1) admits an $\mathbf{S}_{N}$-invariant equilibrium $\varphi_{i j}(t) \equiv C$ for all $C \in \mathbb{R}$, only the choice $C=\Omega(\tau) \tau$ corresponds to an actual $\mathbf{S}_{N}$-invariant equilibrium of the phase model from section 4. 
The matrix $L$ from $(2.6)$ related to the linearization around equilibria $\varphi^{(i, j)}(t)=C$ of the phase-difference model (5.1) is for $N=2$ given by

$$
L=\left(\begin{array}{cccc}
0 & 1 & 0 & 0 \\
-K \mu \cos \left(C+\omega_{M} \tau\right) & -\mu & K \mu \cos \left(C+\omega_{M} \tau\right) e^{-\lambda \tau} & 0 \\
0 & 0 & 0 & 1 \\
K \mu \cos \left(C+\omega_{M} \tau\right) e^{-\lambda \tau} & 0 & -K \mu \cos \left(C+\omega_{M} \tau\right) & -\mu
\end{array}\right)
$$

The characteristic matrix $\triangle(\lambda, \tau):=\lambda I-L$ can be uncoupled into blocks corresponding to isotypic components giving

$$
\rho \triangle \rho^{-1}=\left(\begin{array}{c|c}
\triangle_{1} & 0 \\
\hline 0 & \triangle_{2}
\end{array}\right)
$$

for some transformation $\rho \in \operatorname{Mat}(4)$, and the characteristic functions $P(\lambda, \tau, \eta)=\operatorname{det}(\triangle(\lambda, \tau, \eta))=$ 0 are

$$
P_{1,2}(\lambda, \tau, \eta)=\lambda^{2}+\mu \lambda+K \mu \cos \left(C+\omega_{M} \tau\right) \mp K \mu \cos \left(C+\omega_{M} \tau\right) e^{-\lambda \tau}=0,
$$

which upon substituting $C=\Omega(\tau) \tau$, give the same characteristic functions for the phase model in (4.7) with $N=2$, where $\Omega(\tau) \tau+\omega_{M} \tau=\widehat{\Omega} \tau$.

For a 3-node network using the phase-difference model linearising around the equilibrium point $\varphi^{(i, j)}=\Omega(\tau) \tau$, we obtain

$$
\begin{array}{r}
\triangle(\lambda, \tau)=\left(\lambda^{2}+\mu \lambda\right)^{3}\left(\lambda^{2}+\mu \lambda+K \mu \cos (\widehat{\Omega} \tau)-K \mu \cos (\widehat{\Omega} \tau) e^{-\lambda \tau}\right) \\
\left(\lambda^{2}+\mu \lambda+K \mu \cos (\widehat{\Omega} \tau)+\frac{K \mu}{2} \cos (\widehat{\Omega} \tau) e^{-\lambda \tau}\right)^{2}=0,
\end{array}
$$

and here it is clear that the term $\left(\lambda^{2}+\mu \lambda\right)^{3}$ does not correspond to actual solutions for the characteristic functions for the phase model in (4.7) with $N=3$. 


\section{Chapter 6}

\section{Conclusions and Future work}

\subsection{Conclusions}

In summary, this thesis has the following results.

Due to the $\mathbf{S}_{N}$-symmetry of a second-order $N$-node oscillators network modeled using the full-phase variables, the linearization along every $\mathbf{S}_{N^{-}}$invariant equilibrium can be uncoupled into $N$ blocks, one of them corresponding to the fixed point space $\operatorname{Fix}\left(\mathbf{S}_{N}\right)$, and the others are identical and lead to eigenvalues of multiplicity $N-1$. This decomposition simplifies the bifurcation analysis considerably.

Hopf bifurcation in the first block is symmetry-preserving, i.e. it leads to bifurcation of synchronized periodic orbits which are periodic orbits in $\operatorname{Fix}\left(\mathbf{S}_{N}\right)$; Hopf bifurcations in the other blocks are symmetry-breaking and lead to bifurcation of non-synchronized or partially synchronized periodic orbits.

We presented this decomposition for second order oscillators, but networks of higher order oscillators, modelled accordingly, also have $\mathbf{S}_{N}$-symmetry; therefore, the above mentioned block decomposition also applies provided the time-delay between nodes are the same.

Note that the full-phase model is obtained from the phase model by including the double-frequency term and moving into a comoving frame with velocity $\omega_{M}$. The phasedifference model discussed in section 5 introduces fictitious solutions that may not correspond to real solutions to the phase model analyzed in section 4 even when the equilibrium point is chosen as $\Omega(\tau) \tau$ for $N>2$.

We conclude that of the three models studied here, only the full phase-model from sec- 
tion 3 represents better and without any approximations the dynamics of a fully connected $N$-node time-delay network.

\subsection{Future Work}

We now list some possible extensions of this thesis.

1. While stability of bifurcations and periodic solutions for ODEs is a well established theory, the same problem for DDE's is more delicate, and will be the focus of future work.

2. In this thesis, we chose a classical PLL as oscillator; however, there are many others types of PLLs in the literature; the main difference between them is in the phase detection. It is possible to generalize our results for a generic smooth phase-detector function since the $\mathbf{S}_{N}$-symmetry is preserved. The type and the characteristics of this generic phase detection function will be also the subject of future research. 


\section{Bibliography}

ACEBRÓN, J. A.; BONILlA, L. L.; PÉREZ VICENTE, C. J.; RITORT, F.; SPIGLER, R. The Kuramoto model: A simple paradigm for synchronization phenomena. Rev. Mod. Phys., 77:137-185 (2005). doi:10.1103/RevModPhys.77.137.

ASL, F. M.; ULSOY, A. G. Analysis of a System of Linear Delay Differential Equations. Journal of Dynamic Systems, Measurement, and Control, 125(2):215 (2003). doi:10.1115/1.1568121.

BERETTA, E.; YANG, K. Geometric Stability Switch Criteria in Delay Differential Systems with Delay Dependent Parameters. SIAM Journal on Mathematical Analysis, 33(5):1144-1165 (2002). doi:10.1137/S0036141000376086.

BEST, R. Phase Locked Loops: Design, Simulation, and Applications. McGrawHill Professional (2007). ISBN 0071493751.

BUEnO, A. M.; FERREIRA, A. A.; PIQUeIRA, J. R. C. Fully Connected PLL Networks: How Filter Determines the Number of Nodes. Mathematical Problems in Engineering (2009). doi:10.1155/2009/256765.

BUEnO, A. M.; FERREIRA, A. A.; PIQUEIRA, J. R. C. Modeling and Filtering Double-Frequency Jitter in One-Way Master Slave Chain Networks. Circuits and Systems I: Regular Papers, IEEE Transactions on, 57(12):3104 -3111 (2010). ISSN 1549-8328. doi:10.1109/TCSI.2010.2052514.

COLlERA, J. A. Bifurcations of Periodic Solutions of Functional Differential Equations with Spatio-Temporal Symmetries. Ph.D. thesis, Queen's University Kingston, Ontario, Canada (2012). 
CORLESS, R. M.; GONNET, G. H.; HARE, D. E. G.; JEFFREY, D. J.; KNUTH, D. E. On the Lambert $W$ function. Adv. Comput. Math., 5(4):329-359 (1996). ISSN 1019-7168. doi:10.1007/BF02124750.

CORREA, D. P. F.; PIQUEIRA, J. R. C. Synchronous states in time-delay coupled periodic oscillators: A stability criterion. Communications in Nonlinear Science and Numerical Simulation, 18(8):2142 - 2152 (2013). ISSN 1007-5704. doi:10.1016/j.cnsns. 2012.12.031.

DIAS, A. P. S.; RODRIGUES, A. Hopf bifurcation with $\mathbf{S}_{N^{-}}$-symmetry. Nonlinearity, 22(3):627 (2009).

ENGELBORGHS, K.; LUZYANINA, T.; ROOSE, D. Numerical bifurcation analysis of delay differential equations using DDE-BIFTOOL. ACM Trans. Math. Softw., 28(1):1-21 (2002). ISSN 0098-3500. doi:10.1145/513001.513002.

ENGELBORGHS, K.; LUZYANINA, T.; SAMAEY, G. DDE-BIFTOOL v. 2.00: a Matlab package for bifurcation analysis of delay differential equations. In Numerical Analysis and Applied Mathematics Section. Department of Computer Science, K.U.Leuven, Leuven, Belgium (2001).

FIEDLER, B.; SANDSTEDE, B.; SCHEEL, A.; WULFF, C. Bifurcation from relative equilibria of noncompact group actions: Skew products, meanders, and drifts. Documenta Mathematica, 1:479-505 (1996).

GARDNER, F. M. Phaselock Techniques. 425 pages, John Wiley \& Sons (2005).

GIANNAKOPOULOS, F.; ZAPP, A. Bifurcations in a planar system of differential delay equations modeling neural activity. Physica D: Nonlinear Phenomena, 159(3?4):215 - 232 (2001). ISSN 0167-2789. doi:10.1016/S0167-2789(01)00337-2.

GILSINN, D. E. Bifurcations, center manifolds, and periodic solutions. In Delay differential equations, pages 155-202. Springer, New York (2009).

GOLUBITSKY, M.; STEWART, I. The symmetry perspective. From equilibrium to chaos in phase space and physical space, volume 200 of Progress in Mathematics. xviii +325pp pages, Birkhäuser Verlag, Basel (2002). ISBN 3-7643-6609-5. doi:10.1007/978-3-0348-8167-8. 
GOLUBITSKY, M.; STEWART, I.; SCHAEFFER, D. G. Singularities and groups in bifurcation theory. Vol. II, volume 69 of Applied Mathematical Sciences. xvi+533 pages, Springer-Verlag, New York (1988). ISBN 0-387-96652-8. doi:10.1007/ 978-1-4612-4574-2.

GORECKI, H.; FUKSA, S.; GRABOWSKI, P.; KOTYTOWSKI, A. Analysis and Synthesis of Time Delay Systems. John Wiley \& Sons (1989).

GYŐRI, I.; LADAS, G. Oscillation theory of delay differential equations. Oxford Mathematical Monographs. xii +368 pages, The Clarendon Press Oxford University Press, New York (1991). ISBN 0-19-853582-1. With applications, Oxford Science Publications.

HALE, J. K. Theory of Functional Differential Equations (Applied Mathematical Sciences). Springer (1977). ISBN 0387902031.

KRAWCEWICZ, W.; WU, J. Theory and applications of Hopf bifurcations in symmetric functional-differential equations. Nonlinear Anal., 35(7, Ser. A: Theory Methods):845-870 (1999). ISSN 0362-546X. doi:10.1016/S0362-546X(97)00711-6.

KUANG, Y. Delay differential equations with applications in population dynamics, volume 191 of Mathematics in Science and Engineering. xii +398 pages, Academic Press Inc., Boston, MA (1993). ISBN 0-12-427610-5.

KUDREWICZ, J.; WASOWICZ, S. Equations of Phase Loops Dynamics on Circle, Torus and Cylinder. World Scientific (2007).

LI, W.; YANG, H.; WEN, L.; WANG, K. Global exponential stability for coupled retarded systems on networks: A graph-theoretic approach. Communications in Nonlinear Science and Numerical Simulation, 19(6):1651-1660 (2014). doi:10.1016/j. cnsns.2013.09.039.

MARTINS, A.; MONTEIRO, L. Frequency transitions in synchronized neural networks. Communications in Nonlinear Science and Numerical Simulation, 18(7):17861791 (2013). ISSN 1007-5704. doi:10.1016/j.cnsns.2012.11.018.

MATHEWS, J. H.; FINK, K. K. Numerical Methods Using Matlab (4th Edition). Pearson (2004). ISBN 0130652482. 
MICHIELS, W.; NICULESCU, S.-I. Stability and stabilization of time-delay systems, volume 12 of Advances in Design and Control. xxii +378 pages, Society for Industrial and Applied Mathematics (SIAM), Philadelphia, PA (2007). ISBN 978-0898716-32-0. doi:10.1137/1.9780898718645. An eigenvalue-based approach.

MONTALDI, J. Relative equilibria and conserved quantities in symmetric Hamiltonian systems. In Peyresq lectures on nonlinear phenomena (Peyresq, 1998/1999), pages 239-280. World Sci. Publ., River Edge, NJ (2000). doi:10.1142/ $9789812792778 \_0008$.

MONTEIRO, L. H. A.; DOS SANTOS, R.; PIQUEIRA, J. R. C. Estimating the critical number of slave nodes in a single-chain PLL network. Communications Letters, IEEE, 7(9):449-450 (2003). ISSN 1089-7798. doi:10.1109/LCOMM.2003.817322.

OLIEN, L.; BELAIR, J. Bifurcations, stability, and monotonicity properties of a delayed neural network model. Physica D: Nonlinear Phenomena, 102(3-4):349 363 (1997). ISSN 0167-2789. doi:10.1016/S0167-2789(96)00215-1.

PIQUEIRA, J. R. C. Network of phase-locking oscillators and a possible model for neural synchronization. Communications in Nonlinear Science and Numerical Simulation, 16(9):3844 - 3854 (2011). ISSN 1007-5704. doi:10.1016/j.cnsns.2010.12.031.

PIQUEIRA, J. R. C.; OLIVEIRA, M. Q.; MONTEIRO, L. H. A. Synchronous state in a fully connected phase-locked loop network. Mathematical Problems in Engineering, 2006 (2006). doi:10.1155/MPE/2006/52356.

PIQUEIRA, J. R. C.; ORSATTI, F. M.; MONTEIRO, L. H. A. Computing with phase locked loops: choosing gains and delays. Neural Networks, IEEE Transactions on, 14(1):243 - 247 (2003). ISSN 1045-9227. doi:10.1109/TNN.2002.806633.

RUAN, H.; KRAWCEWICZ, W.; FARZAMIRAD, M.; BALANOV, Z. Applied equivariant degree. part II: Symmetric Hopf bifurcations of functional differential equations. Discrete and Continuous Dynamical Systems, 16(4):923-960 (2006). doi:10.3934/dcds.2006.16.923.

SHINOZAKI, H.; MORI, T. Robust stability analysis of linear time-delay systems 
by Lambert function: Some extreme point results. Automatica, 42(10):1791 - 1799 (2006). ISSN 0005-1098. doi:http://dx.doi.org/10.1016/j.automatica.2006.05.008.

SMITH, H. An introduction to delay differential equations with applications to the life sciences, volume 57 of Texts in Applied Mathematics. xii +172 pages, Springer, New York (2011). ISBN 978-1-4419-7645-1. doi:10.1007/978-1-4419-7646-8.

SONG, Z.-G.; XU, J. Stability switches and double Hopf bifurcation in a twoneural network system with multiple delays. Cogn Neurodyn, 7(6):505-521 (2013). ISSN 1871-4099. doi:10.1007/s11571-013-9254-0.

STROGATZ, S. Exploring complex networks. Nature, 410(6825):268-276 (2001).

VUELLINGS, A.; SCHOELL, E.; LINDNER, B. Spectra of delay-coupled heterogeneous noisy nonlinear oscillators. EUROPEAN PHYSICAL JOURNAL B, 87(2) (2014). ISSN 1434-6028. doi:\{10.1140/epjb/e2014-41064-y\}.

WANG, Z; HU, H. Calculation of the rightmost characteristic root of retarded time-delay systems via Lambert W function. Journal of Sound and Vibration, 318(4-5):757 - 767 (2008). ISSN 0022-460X. doi:http://dx.doi.org/10.1016/j.jsv.2008.04. 052 .

WANG, Z. H. Numerical Stability Test of Neutral Delay Differential Equations. Mathematical Problems in Engineering, 2008:1-11 (2008). doi:10.1155/2008/698043.

WU, J. Symmetry Functional Differential Equations and Neural Networks with Memory. Transactions of the American Mathematical Society, 350(12):4799-4839 (1998). doi:10.1090/S0002-9947-98-02083-2.

YUAN, Y.; ZHAO, X.-Q. Global stability for non-monotone delay equations (with application to a model of blood cell production). Journal of Differential Equations, 252(3):2189-2209 (2012). ISSN 0022-0396. doi:10.1016/j.jde.2011.08.026. 


\section{Appendix A}

\section{The PLL network model}

In the classical approach (see Best, 2007, Gardner, 2005), the PLL is composed of a phase detector $(\mathrm{PD})$, a filter $(F)$ and a VCO as shown in figure A.1.

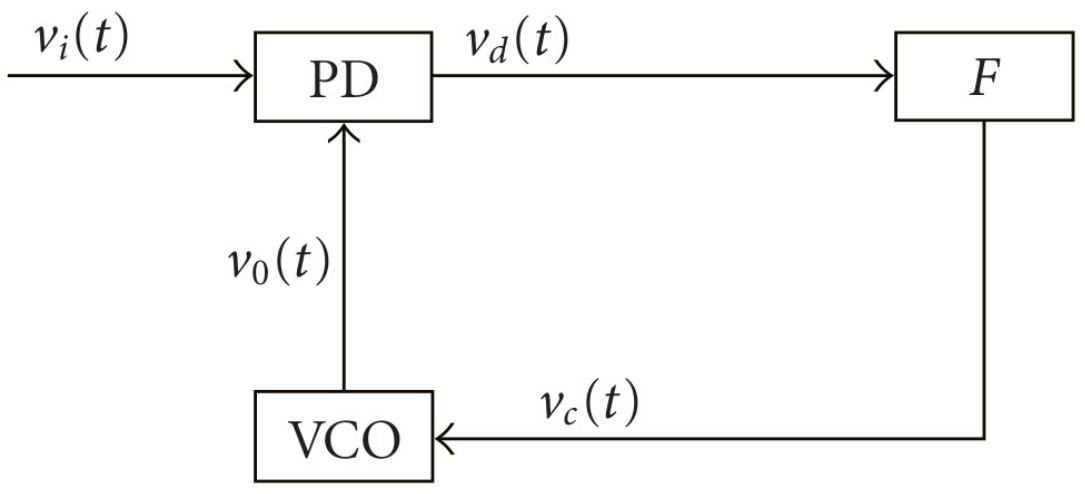

Figure A.1: PLL block diagram

where

- $\nu_{i}(t)$ is the PLL input signal, whose phase is followed by the VCO output phase.

- $\nu_{0}(t)$ is the PLL output signal,

- $\nu_{d}(t)$ is the phase-detector output signal,

- $\nu_{c}(t)$ is the filter output signal.

Considering the arrangement depicted in figure A.2, where each circle represents a PLL, we have that for the PLL 1, the VCO output (also considered as the PLL output) is

$$
\nu_{o}^{(1)}(t)=V \cos \left(\phi_{1}(t)\right)
$$




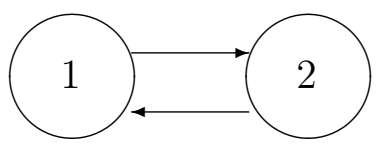

Figure A.2: 2-node network

and,

$$
\phi_{1}(t)=\omega_{1} t+\theta_{1}(t)
$$

$\phi_{1}(t)$ represents the phase of the PLL, called the full-phase, $\omega_{1}$ is the VCO central frequency (also known as free-running frequency), $\theta_{1}(t)$ is an adjustable instantaneous phase which is modified by the output from the filter in order to synchronize $\phi_{1}(t)$ to the input signal phase, and $V$ is the amplitude of the signal.

The input signal is

$$
\nu_{i}^{(1)}(t)=\nu_{o}^{(2)}\left(t-\tau_{21}\right)=V \sin \left(\phi_{2}\left(t-\tau_{21}\right)\right)
$$

$\tau_{21}$ is the time it takes the signal to go from node 2 to node 1 . At a synchronous PLL state, input and output signals are in quadrature; this fact is anticipated in the literature by choosing the input signal proportional to $\sin \left(\phi_{2}(t)\right)$ even when it comes from another PLL output; examples of this can be seen in Acebrón et al. (2005); Bueno et al. (2010); Correa and Piqueira (2013); Gardner (2005); Kudrewicz and Wasowicz (2007); Monteiro et al. (2003); Piqueira et al. (2006, 2003).

The phase detector (PD) is usually modelled as a multiplier

$$
\nu_{d}^{(1)}(t)=k_{m} \nu_{0}^{(2)}\left(t-\tau_{21}\right) \nu_{0}^{(1)}(t)
$$

$k_{m}$ is a scalar gain. Then substituting $(\mathrm{A} .1)$ and $(\mathrm{A} .3)$ into $\mathrm{A} .4$ we obtain

$$
\nu_{d}^{(1)}(t)=\frac{k_{m} V^{2}}{2}\left[\sin \left(\phi_{2}\left(t-\tau_{21}\right)-\phi_{1}(t)\right)+\sin \left(\phi_{2}\left(t-\tau_{21}\right)+\phi_{1}(t)\right)\right] .
$$

For a second-order PLL, the relationship between $\nu_{d}(t)^{(1)}$ and $\nu_{c}(t)^{(1)}$ in the filter is given by

$$
\dot{\nu}_{c}^{(1)}(t)+\mu \nu_{c}^{(1)}(t)=\mu \nu_{d}^{(1)}(t)
$$

$\mu$ is a control parameter in the filter; a generalization of this relationship for higher order PLLs is presented by Bueno et al. (2010). The usually accepted VCO model is given by 
the relation (Gardner, 2005),

$$
\nu_{c}^{(1)}(t)=\frac{\dot{\theta}_{1}(t)}{k_{0}}
$$

$k_{0}$ is a gain control parameter, then substituting $\mathrm{A} .2 \mathrm{n}$ in the filter output we have

$$
\nu_{c}^{(1)}(t)=\frac{1}{k_{0}}\left(\dot{\phi}_{1}(t)-\omega_{1}\right)
$$

then, substituting equations A.7 and A.5 into A.6), we obtain

$$
\ddot{\phi}_{1}(t)+\mu \dot{\phi}_{1}(t)-\mu \omega_{1}-K \mu\left[\sin \left(\phi_{2}\left(t-\tau_{21}\right)-\phi_{1}(t)+\sin \left(\phi_{2}\left(t-\tau_{21}\right)+\phi_{1}(t)\right)\right]=0,\right.
$$

here

$$
K:=\frac{1}{2} k_{0} k_{m} V^{2}
$$

is called the PLL gain. Thus the dynamics for the 2-node network depicted in A.2 is given

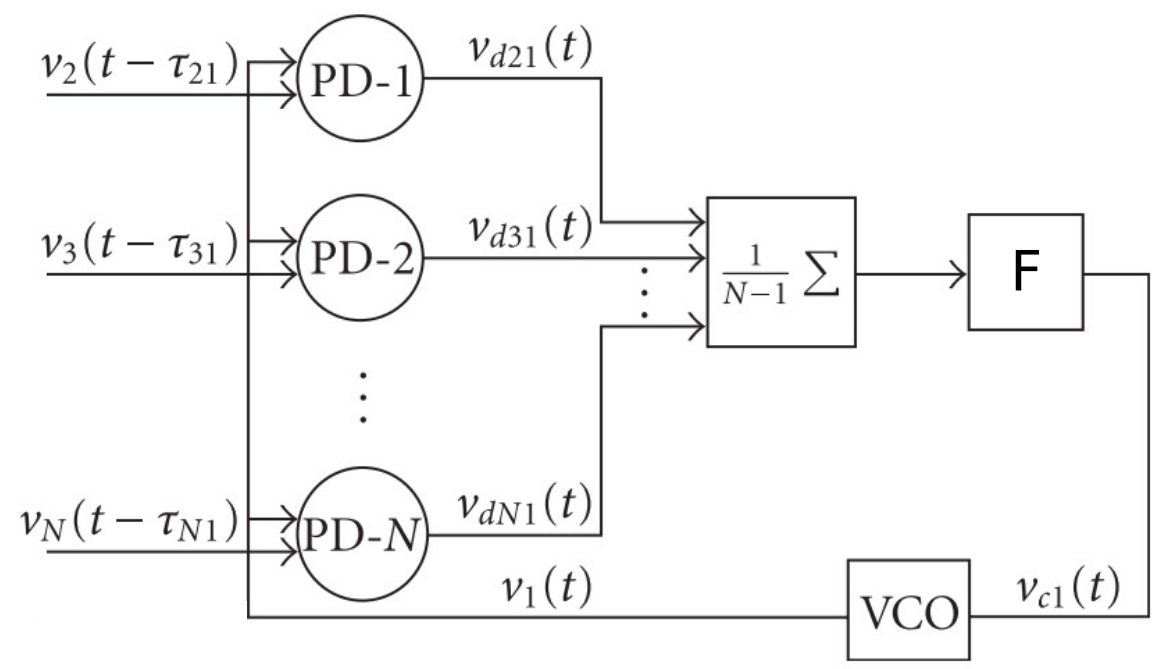

Figure A.3: PLL with multiple inputs.

by the system of second-order coupled RFDEs

$$
\begin{aligned}
& \ddot{\phi}_{1}(t)+\mu \dot{\phi}_{1}(t)-\mu \omega_{1}-K \mu\left[\sin \left(\phi_{2}\left(t-\tau_{21}\right)-\phi_{1}(t)+\sin \left(\phi_{2}\left(t-\tau_{21}\right)+\phi_{1}(t)\right)\right]=0\right. \\
& \ddot{\phi}_{2}(t)+\mu \dot{\phi}_{2}(t)-\mu \omega_{2}-K \mu\left[\sin \left(\phi_{1}\left(t-\tau_{12}\right)-\phi_{2}(t)+\sin \left(\phi_{1}\left(t-\tau_{12}\right)+\phi_{2}(t)\right)\right]=0,\right.
\end{aligned}
$$

For an N-node fully connected network, where each node has the structure shown in figure A.3 and considering all identical nodes, i.e., $\omega_{1}=\ldots=\omega_{N}=\omega_{M}$, and identical all 
the time delays between nodes, i.e., $\tau_{12}=\ldots=\tau_{1 N}=\tau_{21}=\ldots=\tau$, for the $i$-th node we obtain (see Piqueira et al., 2006)

$$
\begin{aligned}
& \ddot{\phi}_{i}(t)+\mu \dot{\phi}_{i}(t) \\
& -\mu \omega_{M}-\frac{K \mu}{N-1} \sum_{\substack{j=1 \\
j \neq i}}^{N}\left[\sin \left(\phi_{j}(t-\tau)-\phi_{i}(t)+\sin \left(\phi_{j}(t-\tau)+\phi_{i}(t)\right)\right]=0,\right.
\end{aligned}
$$

which has equilibria at

$$
\begin{aligned}
& 2 \phi^{+}=\arcsin \left(-\frac{\omega_{M}}{K}\right)+2 k \pi, \\
& 2 \phi^{-}=\pi-\arcsin \left(-\frac{\omega_{M}}{K}\right)+2 k \pi,
\end{aligned}
$$

